\title{
Polaron-cyclotron-resonance spectrum resulting from interface- and slab-phonon modes in a GaAs/AlAs quantum well
}

\author{
G. Q. Hai, F. M. Peeters, and J. T. Devreese* \\ Departement Natuurkunde, Universiteit Antwerpen (UIA), Universiteitsplein 1, B-2610 Antwerpen, Belgium
}

(Received 10 December 1992)

\begin{abstract}
The effects of interface optical-phonon and confined slab LO-phonon modes on the polaron cyclotron-resonance frequency are investigated for a GaAs/AlAs quantum well. Using degenerate second-order perturbation theory, the polaron Landau levels are calculated and the polaron resonant region is investigated. In order to know the relative importance of the different resonant frequencies we present a full calculation of the magneto-optical absorption spectrum. At a fixed magnetic field we found four different peaks in the absorption spectrum. The relative oscillator strength of the different peaks changes with increasing magnetic field. For comparative purposes, the polaron Landau levels and cyclotron mass are also calculated using only the bulk LO-phonon modes. The influence of the finiteness of the confinement potential is investigated. We found that the interface-phonon modes influence the magnetopolaron resonance considerably near the optical-phonon frequencies for narrow wells. In the limit of zero magnetic field we recover our previous results and in the case of an infinite-barrier quantum well we are able to recover the results for a two- and three-dimensional system.
\end{abstract}

\section{INTRODUCTION}

Polarons in polar semiconductors and ionic crystals have been extensively studied both theoretically and experimentally. ${ }^{1}$ In the presence of a magnetic field the socalled resonant-polaron effect occurs. ${ }^{2-12}$ These studies show that the Landau levels are modified by the electron-LO-phonon interaction in the following way: (i) they are shifted to lower energy, (ii) the slope of the Landau levels versus the magnetic field is changed, (iii) the unperturbed Landau levels split near the LO-phonon energy, and, (iv) in high magnetic fields the lower branch of the split Landau levels is pinned to the LO-phonon branch. In cyclotron-resonance experiments ${ }^{7-11}$ on bulk materials the mass renormalization of the electron due to polaron effects can be observed.

Recent developments in the semiconductor technology have made it possible to create quasi-two-dimensional (Q2D) electron systems. For fundamental research the most important systems are based on $\mathrm{GaAs} / \mathrm{Al}_{x} \mathrm{Ga}_{1-x} \mathrm{As}$ heterojunctions, quantum wells, and superlattices because of the low disorder. In the last decade, polaron effects in two-dimensional (2D) systems ${ }^{13-33}$ have received considerable attention. Theoretical investigations, using bulk LO-phonon modes, show that the behavior of the polaron Landau levels and the cyclotron mass in a $2 \mathrm{D}$ system is qualitatively very similar to the three-dimensional (3D) equivalent. But it was found that polaron effects are enhanced in a $2 \mathrm{D}$ system. Several scaling relations between the quantities in $2 \mathrm{D}$ and in $3 \mathrm{D}$ have been derived by Peeters and Devreese. ${ }^{18}$ Later studies have found that, in a real 2D system, the electron-phonon interaction is reduced by many-body effects. ${ }^{14,24}$

In all the above works, it was assumed that the LOphonon modes were not influenced by the presence of the interfaces. And most of the theories are for single semiconductor heterojunction structures. On the other hand, we know that in a Q2D semiconductor structure the phonon modes are modified by the presence of the interfaces. Recently, the optical-phonon modes in a Q2D semiconductor-structure system have been studied ${ }^{34-38}$ and the electron-phonon interaction Hamiltonian was derived.

In the absence of a magnetic field, several theoretical results have been obtained on polarons in Q2D systems ${ }^{39-44}$ where interface- and slab-phonon modes are included. Only for very narrow quantum wells are the results quantitatively different from the bulk-phonon case. In the presence of a magnetic field, $\mathrm{Gu}, \mathrm{Kong}$, and $\mathrm{Wei}^{45}$ and Liang ${ }^{46}$ have calculated the cyclotron frequency of a polaron in a polar slab in weak magnetic field with surface- and slab-phonon modes. Gu, Kong, and Wei ${ }^{47}$ studied the polaron Landau levels in a quantum well, where only the lowest Landau level associated with the first two electric subbands are calculated. Lin, Chen, and George $^{48}$ studied the bound-polaron resonance in a GaAs $/ \mathrm{Al}_{x} \mathrm{Ga}_{1-x}$ As quantum well. Their result shows that, due to the interface phonons, the $1 s-2 p^{+}$transition of a bound polaron is pinned to the TO-phonon frequency in the limit of high magnetic fields in a $125-\AA$ quantum well. The present investigation does not support this conclusion.

In the present paper, we report an investigation of the polaron Landau levels and cyclotron-resonance spectrum in a quantum well as induced by the electron-opticalphonon interaction. The interface optical-phonon and confined slab LO-phonon modes, as well as the 3D bulk LO-phonon modes, will be incorporated in our calculations.

We will choose a GaAs/AlAs quantum well as our calculation model. The confinement potential of the quantum well will be assumed along the $z$ direction, and the interface of the quantum well in the $x y$ plane. A magnetic field is applied perpendicular to the interface. We 
denote GaAs as material 1 and AlAs as material 2. The material parameters ${ }^{49}$ are given in Table $I$ in Ref. 44. The conduction-band mass of GaAs $m_{b 1}=0.067 m_{0}$, where $m_{0}$ is the electron mass in vacuum. The LOphonon frequency of GaAs will be denoted by $\omega_{\mathrm{LO}}$. Dimensionless units are used such that $\hbar=\omega_{\mathrm{LO}}=m_{b 1}=1$.

Theoretically there are two different viewpoints in calculating the cyclotron-resonance frequency. One is by starting from the position of the Landau levels, and the cyclotron frequency is given by the difference between the Landau levels. Another is by calculating the magnetooptical absorption spectrum, and the cyclotron frequency is determined by the position of the peaks in the absorption spectrum. In the present paper, we will calculate both the polaron Landau levels and the cyclotronresonance spectrum. First, we will calculate the polaron Landau levels using degenerate second-order perturbation theory. Interaction with interface- and slab-phonon modes will be included. We will compare our results with the case where the electron interacts with 3D LOphonon modes. Second, we present a calculation of the magneto-optical absorption spectrum from which we will obtain the cyclotron-resonance frequency and the oscillator strength of the different peaks. This will allow us to know the relative importance of the different peaks in the spectrum.

The present paper is organized as follows. The Hamiltonian for the coupling of an electron to the optical phonons in a GaAs/AlAs quantum well is given in Sec. II. In Sec. III the polaron Landau levels in such a system will be calculated using degenerate second-order perturbation theory, from which we obtain the cyclotron frequency. 3D LO-phonon modes and the interface and confined slab LO-phonon modes are considered. Two different confinement potentials, infinite- and finite-height barrier quantum wells, are used in order to investigate the influence of the confinement potential on our results. In Sec. IV the magneto-optical absorption spectrum and the related memory function with the interface- and slab-phonon modes will be calculated. Our conclusions are presented in Sec. V.

\section{THE HAMILTONIAN}

For completeness we give a short review of the Hamiltonian. The system under consideration is described by the following Hamiltonian:

$$
H=H_{e}+H_{\mathrm{ph}}+H_{e p}
$$

with

$$
\begin{aligned}
& H_{e}=\frac{p_{x}^{2}}{2 m_{b}}+\frac{1}{2 m_{b}}\left(p_{y}+e B x\right)^{2}+\frac{p_{z}^{2}}{2 m_{b}}+V(z), \\
& H_{\mathrm{ph}}=\sum_{\mathrm{q}} \hbar \omega_{\mathrm{q}}\left(a_{\mathrm{q}}^{\dagger} a_{\mathrm{q}}+\frac{1}{2}\right)
\end{aligned}
$$

where $B$ is the magnetic field directed along the $z$ axis, $\mathbf{p}$ (r) the momentum (position) operator of the electron, $V(z)$ the confinement potential of the quantum well, $a_{q}^{\dagger}$ $\left(a_{\mathrm{q}}\right)$ the creation (annihilation) operator of an optical phonon with wave vector $q$ and energy $\hbar \omega_{q}$, and $m_{b}$ the electron-band mass which is given by

$$
m_{b}= \begin{cases}m_{b 1}, & |z|<W / 2 \\ m_{b 2}, & |z|>W / 2\end{cases}
$$

where $m_{b 1}$ and $m_{b 2}$ are the electron-band mass of GaAs and AlAs, respectively, and $W$ is the width of the quantum well.

For an electron in a Q2D system interacting with 3D bulk LO-phonon modes, the electron-phonon interaction Hamiltonian $H_{e p}$ in Eq. (1) is given by the Fröhlich interaction Hamiltonian

$$
H_{e p}=\sum_{\mathbf{q}}\left(V_{\mathbf{q}} a_{\mathbf{q}} e^{i \mathbf{q} \cdot \mathbf{r}}+V_{\mathbf{q}}^{*} a_{\mathbf{q}}^{\dagger} e^{-i \mathbf{q} \cdot \mathbf{r}}\right)
$$

where

$$
V_{\mathrm{q}}=-i \hbar \omega_{\mathrm{LO}}\left(\frac{\hbar}{2 m_{b 1} \omega_{\mathrm{LO}}}\right)^{1 / 4} \sqrt{4 \pi \alpha_{1} / V q^{2}}
$$

and $\alpha_{1}$ is the coupling constant of the material inside the quantum well.

In a semiconductor heterostructure the phonon modes are modified because of the presence of the interfaces. For a single GaAs/AlAs quantum-well structure, there are four types of optical-phonon modes interacting with the electrons: (1) symmetric interface optical-phonon modes with frequency $\omega_{S \pm}\left(q_{\|}\right)$, (2) antisymmetric interface optical-phonon modes with frequency $\omega_{A \pm}\left(q_{\|}\right)$, (3) confined slab LO-phonon modes in the well with frequency $\omega_{L 1}$, and (4) half-space LO-phonon modes in the barrier layers with frequency $\omega_{L 2}$.

The dispersion relation for the interface phonons is given by ${ }^{34,35,44}$

$$
\begin{aligned}
\omega_{I, \pm}\left(q_{\|}\right)=\left\{\frac{1}{2\left(\epsilon_{1}^{I}+\epsilon_{2}^{I}\right)}(\right. & \epsilon_{1}^{I}\left(\omega_{L 1}^{2}+\omega_{T 2}^{2}\right)+\epsilon_{2}^{I}\left(\omega_{L 2}^{2}+\omega_{T 1}^{2}\right) \\
& \left.\left. \pm\left\{\left[\epsilon_{1}^{I}\left(\omega_{L 1}^{2}+\omega_{T 2}^{2}\right)+\epsilon_{2}^{I}\left(\omega_{L 2}^{2}+\omega_{T 1}^{2}\right)\right]^{2}-4\left(\epsilon_{1}^{I}+\epsilon_{2}^{I}\right)\left(\epsilon_{1}^{I} \omega_{L 1}^{2} \omega_{T 2}^{2}+\epsilon_{2}^{I} \omega_{L 2}^{2} \omega_{T 1}^{2}\right)\right\}^{1 / 2}\right)\right\}^{1 / 2},
\end{aligned}
$$




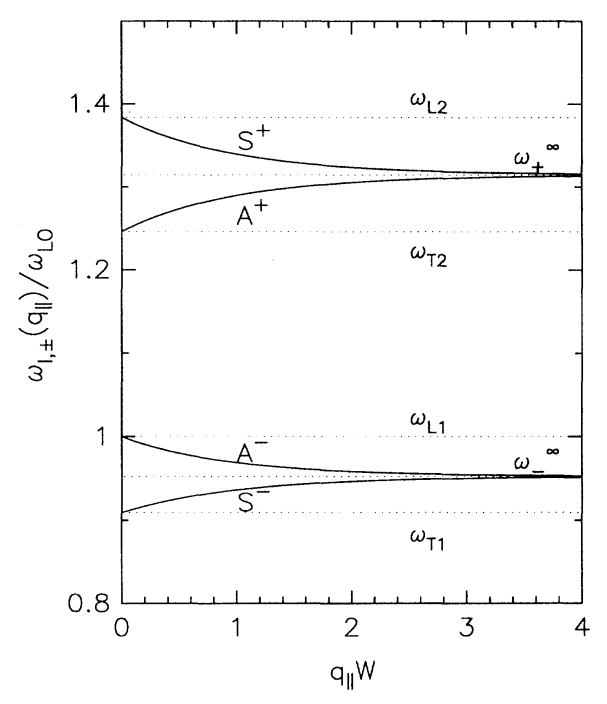

FIG. 1. The dispersion relations of the interface-phonon modes in a GaAs/AlAs quantum-well structure.

where $\epsilon_{1}^{I}=\epsilon_{\infty 1}\left(1-\gamma_{I} e^{-q_{\|} W}\right), \epsilon_{2}^{I}=\epsilon_{\infty 2}\left(1+\gamma_{1} e^{-q_{\|} W}\right)$, and $I=S, A$ refers to the symmetric $(S)$ and antisymmetric ( $A$ ) modes, respectively, $\gamma_{S}=1, \gamma_{A}=-1, \quad \omega_{L v}^{2}$ $=\omega_{T v}^{2}\left(\epsilon_{0 v} / \epsilon_{\infty v}\right), v=1,2$. In Fig. 1 the dispersion curves of the interface-phonon modes in a GaAs/AlAs quantum-well structure are plotted. It is seen that $\omega_{S,-}=\omega_{T 1}, \omega_{A,-}=\omega_{L 1}, \omega_{A,+}=\omega_{T 2}$, and $\omega_{S,+}=\omega_{L 2}$ at $q_{\|}=0$. When $q_{\|}$goes to infinity, $\omega_{I,+}\left(q_{\|} \rightarrow \infty\right)=\omega_{+}^{\infty}$, $\omega_{I,-}\left(q_{\|} \rightarrow \infty\right)=\omega_{-}^{\infty}$. The $(-)$ modes occur in the reststrahlen branch of GaAs, the $(+)$ modes in that of AlAs.
The confined slab LO-phonon modes are dispersionless and with frequency $\omega_{L 1}$, but their momenta in the $z$ direction are quantized and $q_{z}=j \pi / W, j$ $=1,2,3, \ldots, j_{\max }$, where $j_{\max }=\operatorname{int}\left(W / a_{0}\right), a_{0}=5.65 \AA$ is the lattice constant of GaAs.

The electron-phonon interaction Hamiltonian can be written in the following form: ${ }^{34,35,44}$

$$
H_{e p}=\sum_{j} \sum_{\mathbf{q}_{\|}} e^{i \mathbf{q}_{\|} \cdot \mathbf{r}_{\|}} \Gamma_{j}\left(\mathbf{q}_{\|}, z\right)\left[a_{j}\left(\mathbf{q}_{\|}\right)+a_{j}^{\dagger}\left(-\mathbf{q}_{\|}\right)\right],
$$

where $\Gamma_{j}\left(\mathbf{q}_{\|}, z\right)$ is the coupling function that describes the coupling strength of a single electron at the position $z$ with the $j$ th optical-phonon mode with the dispersion relation $\omega_{j}\left(q_{\|}\right)$. The expression of the coupling function was given by Eqs. (13)-(17) in Ref. 44.

\section{THE POLARON LANDAU LEVELS}

The state of a bare electron in such a system is determined by the Hamiltonian $H_{e}$. The eigenenergy of the bare electron is

$$
E_{n, l}^{0}=\hbar \omega_{c}\left(n+\frac{1}{2}\right)+E_{l}^{z},
$$

where $E_{l}^{z}$ is the electric level in the $z$ direction, $n$ is the Landau level index, $\omega_{c}=e B / m_{c}$ is the unperturbed cyclotron frequency, and $m_{c}$ is given by

$$
m_{c}=\frac{m_{b 1} m_{b 2}}{P_{w} m_{b 2}+P_{b} m_{b 1}}
$$

where $P_{w}\left(P_{b}\right)$ is the probability to find the electron inside (outside) the quantum well. The corresponding wave function can be written as

$$
\Psi_{n, k_{y}, l}(x, y, z)=\left[\frac{m_{c} \omega_{c}}{\pi \hbar}\right]^{1 / 4} \frac{1}{\sqrt{2^{n} n !}} H_{n}\left(\sqrt{m_{c} \omega_{c} / \hbar x^{\prime}}\right) \exp \left(\frac{-m_{c} \omega_{c} x^{\prime 2}}{2 \hbar}\right) \frac{1}{\sqrt{L_{y}}} e^{i k_{y} y} \psi_{l}(z)
$$

where $x^{\prime}=x+\hbar k_{y} / m_{c} \omega_{c}, H_{n}(x)$ are the Hermite polynomials, and $\psi_{l}(z)$ is the wave function in the $z$ direction as determined by $V(z)$ in the electric subband $l$.

For an infinite-barrier square well, one has

$$
\begin{aligned}
& E_{l}^{z}=\frac{\hbar^{2} \pi^{2}}{2 m_{b 1} W^{2}} l^{2}, \quad l=1,2,3, \ldots, \\
& \psi_{l}(z)=\sqrt{2 / W} \sin [(z+W / 2) l \pi / W],
\end{aligned}
$$

for $|z|<W / 2$, and $\psi_{l}(z)=0$ for $|z|>W / 2$. Consequently, $P_{w}=1, P_{b}=0$, and $m_{c}=m_{b 1}$.

And for a finite-barrier square well, the confinement potential can be expressed in the following form:

$$
V(z)=\left\{\begin{array}{l}
0, \quad|z|<W / 2 \\
V_{0}, \quad|z|>W / 2
\end{array}\right.
$$

where $V_{0}=0.915 \mathrm{eV}$ for a GaAs/AlAs quantum well. The lowest electric level $E_{1}^{z}$ is determined by the equation

$$
\tan \left(\frac{W}{2} \frac{\sqrt{2 m_{b 1} E_{1}^{z}}}{\hbar}\right)=\left(\frac{m_{b 1}\left(V_{0}-E_{1}^{z}\right)}{m_{b 2} E_{1}^{z}}\right)^{1 / 2},
$$

and the wave function is given by

$\psi_{1}(z)=\left\{\begin{array}{l}\boldsymbol{B}_{0} \cos (k z), \quad|z| \leq W / 2 \\ \boldsymbol{B}_{0} \cos (k W / 2) e^{-k_{1}(|z|-W / 2)}, \quad|z|>W / 2,\end{array}\right.$

where $k=\sqrt{2 m_{b 1} E_{1}^{z}} / \hbar, k_{1}=\sqrt{2 m_{b 2}\left(V_{0}-E_{1}^{z}\right)} / \hbar$, and the normalization constant 
$B_{0}=\left(\frac{2 k}{k W+\sin (k W)+2 k \cos ^{2}(k W / 2) / k_{1}}\right)^{1 / 2}$.

In the following, we will calculate the polaron corrections $\Delta E_{n, l}$ to the unperturbed energy levels $E_{n, l}^{0}$ due to the electron-phonon interaction. Then the polaron energy is given by

$$
E_{n, l}=E_{n, l}^{0}+\Delta E_{n, l} .
$$

Based on second-order perturbation theory, the shift of the Landau level in a quantum well due to the electronphonon interaction is given by

$$
\Delta E_{n, l}=-\sum_{n^{\prime}} \sum_{l^{\prime}} \sum_{\mathbf{q}} \frac{\left|M_{n l, n^{\prime} l^{\prime}}(\mathbf{q})\right|^{2}}{\hbar \omega_{\mathbf{q}}-\Delta_{n, l}+\left(n^{\prime}-n\right) \hbar \omega_{c}+E_{l^{\prime}}^{z}-E_{l}^{z}},
$$

where $\Delta_{n, l}=\Delta E_{n, l}-\Delta E_{0,1}$ within the improved WignerBrillouin perturbation theory ${ }^{10}$ (IWBPT), and

$$
M_{n l, n^{\prime} l^{\prime}}(\mathbf{q})=\left\langle n^{\prime}, l^{\prime} ; \mathbf{q}\left|H_{e p}\right| n, l ; 0\right\rangle
$$

is the matrix element of the electron-phonon interaction $H_{e p}$, the ket $|n, l ; \mathbf{q}\rangle=|n\rangle_{\otimes}|l\rangle_{\otimes}|\mathbf{q}\rangle$ describes a state composed of an electron in Landau level $n$ and electric level $l$ and an optical phonon with momentum $\hbar q$ and energy $\hbar \omega_{\mathbf{q}}$.

\section{A. 3D bulk LO-phonon modes}

In this case, the phonon modes in the system are assumed to be the 3D LO-phonon modes of GaAs; the interaction matrix element in Eq. (20) reduces to

$$
\left|\boldsymbol{M}_{n l, n^{\prime} l^{\prime}}(\mathbf{q})\right|^{2}=\left|V_{\mathbf{q}}\right|^{2}\left|\left\langle n\left|e^{i \mathbf{q}_{\|} \cdot \mathbf{r}^{\|} \|}\right| n^{\prime}\right\rangle\right|^{2}\left|G_{l, l^{\prime}}\left(q_{z}\right)\right|^{2}
$$

with

$$
\left|G_{l, l^{\prime}}\left(q_{z}\right)\right|^{2}=\left|\left\langle l\left|e^{i q_{z} z}\right| l^{\prime}\right\rangle\right|^{2}
$$

and

$$
\left|\left\langle n\left|e^{i \mathbf{q}_{\|} \cdot \mathbf{r}_{\|}}\right| n^{\prime}\right\rangle\right|^{2}=\frac{m !}{m^{\prime} !} \xi_{\|}^{m^{\prime}-m} e^{-\xi_{\|}}\left[L_{m}^{m^{\prime}-m}\left(\xi_{\|}\right)\right]^{2},
$$

where $\quad \xi_{\|}=\hbar q_{\|}^{2} /\left(2 m_{c} \omega_{c}\right), \quad m=\min \left(n, n^{\prime}\right)$, $m^{\prime}=\max \left(n, n^{\prime}\right)$, and $L_{m}^{m^{\prime}-m}\left(\xi_{\|}\right)$are the Laguerre polynomials.

Then the polaron correction $\Delta E_{n, l}$ is given by

$$
\Delta E_{n, l}=-\frac{\alpha_{1}}{\sqrt{2} \pi} \sum_{l^{\prime}=1}^{\infty} \sum_{n^{\prime}=0}^{\infty} \frac{m !}{m^{\prime} !} \int_{-\infty}^{\infty} d q_{z} \int_{0}^{\infty} d q_{\|} \frac{q_{\|} \xi_{\|}^{m^{\prime}-m_{e}} e^{-\xi_{\|}}\left[L_{m}^{m^{\prime}-m^{\prime}}\left(\xi_{\|}\right)\right]^{2}\left|G_{l, l^{\prime}}\left(q_{z}\right)\right|^{2}}{\left(q_{\|}^{2}+q_{z}^{2}\right)\left[1-\Delta_{n, l}+\left(n^{\prime}-n\right) \omega_{c}+E_{l}^{\prime z}-E_{l}^{z}\right]} .
$$

For an infinite-barrier quantum well, we obtain

$$
\left|G_{l, l^{\prime}}\left(q_{z}\right)\right|^{2}=\left[\frac{8 l l^{\prime} \pi^{2} q_{z} W}{\left[\left(l-l^{\prime}\right)^{2} \pi^{2}-\left(q_{z} W\right)^{2}\right]\left[\left(l+l^{\prime}\right)^{2} \pi^{2}-\left(q_{z} W\right)^{2}\right]}\right]^{2} \times \begin{cases}\sin ^{2}\left(q_{z} W / 2\right), & l+l^{\prime}=\text { even } \\ \cos ^{2}\left(q_{z} W / 2\right), & l+l^{\prime}=\text { odd } .\end{cases}
$$

For a finite-barrier quantum well, only the leading term $l^{\prime}=l=1$ in the sum over $l^{\prime}$ is considered in Eq. (24); in this way we have

$$
\begin{aligned}
G_{1,1}\left(q_{z}\right)=B_{0}^{2}\{ & 2 \cos ^{2}(k W / 2) \frac{2 k_{1} \cos \left(q_{z} W / 2\right)-q_{z} \sin \left(q_{z} W / 2\right)}{4 k_{1}^{2}+q_{z}^{2}}+\frac{\sin \left[\left(2 k-q_{z}\right) W / 2\right]}{2\left(2 k-q_{z}\right)}+\frac{\sin \left[\left(2 k+q_{z}\right) W / 2\right]}{2\left(2 k+q_{z}\right)} \\
& \left.+\frac{\sin \left(q_{z} W / 2\right)}{q_{z}}\right\} .
\end{aligned}
$$

Equation (24) can be used to calculate the polaron energy $\Delta E_{n, l}$. But in small magnetic field, the sum over $n^{\prime}$ converges so slowly that it is almost impossible to obtain the correct $B \rightarrow 0$ limit results for $\Delta E_{n, l}$. To avoid this defect, we cast this sum into an integration representation as what was done in Ref. 20. For the energy below the LO-phonon continuum, i.e., $E<E_{0,1}+\hbar \omega_{\text {LO }}, \Delta E_{n, l}$ becomes

$$
\begin{aligned}
\Delta E_{n, l}=-\frac{\alpha_{1}}{\sqrt{2} \pi} \int_{0}^{\infty} d u e^{-\left(\omega_{\mathrm{LO}}-\Delta_{n l}-n \omega_{c}\right) u} \sum_{n^{\prime}=0}^{n} & {\left[\begin{array}{c}
n \\
n^{\prime}
\end{array}\right) \frac{1}{n^{\prime} !} e^{-\left(n-n^{\prime}\right) \omega_{c} u}\left(1-e^{-\omega_{c} u}\right)^{n^{\prime}} } \\
& \times \int_{0}^{\infty} d q_{z} I_{n^{\prime}}\left(\mu q_{z}^{2}\right) \sum_{l^{\prime}=1}^{\infty}\left|G_{l, l^{\prime}}\left(q_{z}\right)\right|^{2} e^{-\left(E_{l^{\prime}}^{z}-E_{l}^{z}\right) u},
\end{aligned}
$$


where

$$
\begin{aligned}
& \mu=\frac{\hbar}{2 m_{c} \omega_{c}}\left(1-e^{-\omega_{c} u}\right), \\
& I_{n}(t)=(-t)^{n} I_{0}(t)+\sum_{j=0}^{n-1}(-t)^{j}(n-1-j) !,
\end{aligned}
$$

and

$$
I_{0}(t)=-e^{t} E i(-t) .
$$

$E i(-t)$ is the exponential-integral function. Note, $\hbar \omega_{\mathrm{LO}}-\Delta_{n, l}-n \hbar \omega_{c}>0$ for the energies below the LOphonon continuum, i.e., the denominator in Eq. (24) is larger than zero for all $n^{\prime}$.

For energies above the LO-phonon continuum, the denominator in Eq. (24) may change sign when $n \geq 1$ and the above procedure cannot be used. Nevertheless, the first important region above the LO-phonon continuum satisfies $E_{0,1}+\hbar \omega_{\mathrm{LO}}+\hbar \omega_{c}>E>E_{0,1}+\hbar \omega_{\mathrm{LO}}$, and part of the terms in the sum over $n^{\prime}$ in Eq. (24) can also be cast into an integration representation. After some tedious algebra, we obtain for $n \geq 1$

$$
\begin{aligned}
& \Delta E_{n, l}=-\frac{\alpha_{1}}{\sqrt{2} \pi}\left\{\int_{0}^{\infty} d q_{z} I_{n}\left(\xi_{z}\right)^{n-1} \sum_{j=0}^{n}\left(\begin{array}{c}
n-1 \\
j
\end{array}\right) \frac{(-1)^{j}}{n !} \sum_{l^{\prime}=0}^{\infty} \frac{\left|G_{l, l^{\prime}}\left(q_{z}\right)\right|^{2}}{1-\Delta_{n, l}-(n-j) \omega_{c}+E_{l^{\prime}}^{z}-E_{l}^{z}}\right. \\
& +\int_{0}^{\infty} d u e^{-\left[1-\Delta_{n, l}-(n-1) \omega_{c}\right] u}
\end{aligned}
$$

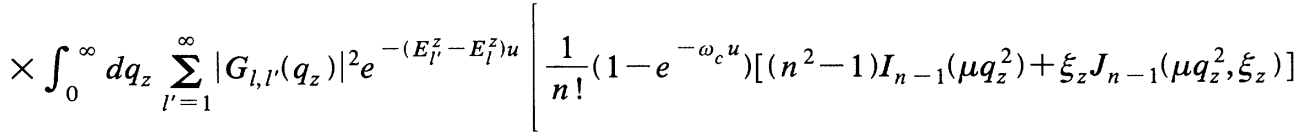

$$
\begin{aligned}
& \left.\left.+\sum_{j=0}^{n-2}\left[\begin{array}{l}
n \\
j
\end{array}\right] e^{-(n-1-j) \omega_{c} u}\left(1-e^{-\omega_{c} u}\right)^{j} I_{j}\left(\mu q_{z}^{2}\right)\right]\right\}
\end{aligned}
$$

where $\xi_{z}=\hbar q_{z}^{2} /\left(2 m_{c} \omega_{c}\right), I_{n}(t)$ is given by Eq. (29), and

$$
J_{n}\left(t_{1}, t_{2}\right)=\frac{I_{n}\left(t_{1}\right)-I_{n}\left(t_{2}\right)}{t_{2}-t_{1}}
$$

In Fig. 2 we show the Landau-level energy $E_{n, 1}=E_{n, 1}^{0}+\Delta E_{n, 1}$ as a function of the magnetic field for $n=0,1,2$, and 3 for a GaAs quantum well of width $W=100 \AA$. In the calculation the infinite-barrier model is used and all intermediate Landau levels and electric energy levels are taken into account. The thin solid lines are the unperturbed energy $E_{n, 1}^{0}$ and the thin dashed lines are $E_{0,1}+\hbar \omega_{\mathrm{LO}}+n \hbar \omega_{c}$. Notice also the pinning of the Landau levels for $B \rightarrow \infty$.

\section{B. Interface- and slab-phonon modes}

The electron-phonon interaction Hamiltonian with interface and confined slab optical phonons is described by Eq. (8), and the corresponding matrix element of electron-phonon interaction in Eq. (20) can be written as

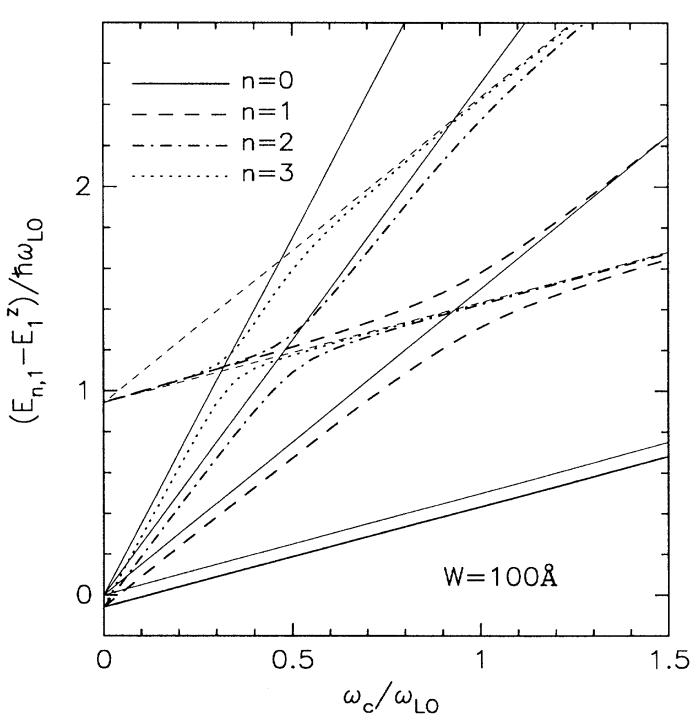

FIG. 2. The first four Landau levels of a polaron in 100- $\AA$ infinite-barrier quantum wells with $3 \mathrm{D}$ LO phonons. The thin solid lines represent the unperturbed Landau levels. The thin dashed lines indicate the energy levels $E_{0,1}+\hbar \omega_{\mathrm{LO}}+n \hbar \omega_{c}$. 
$\left|M_{n l, n^{\prime} l^{\prime}}(\mathbf{q})\right|^{2}=\left|\left\langle l\left|\Gamma_{j}\left(\mathbf{q}_{\|}, z\right)\right| l^{\prime}\right\rangle\right|^{2}\left|\left\langle n\left|e^{i \mathbf{q}_{\|} \cdot \mathbf{r}_{\|}}\right| n^{\prime}\right\rangle\right|^{2}$,

where $\left|\left\langle n\left|e^{i \mathbf{q}_{\|} \cdot \mathbf{r}_{\|}}\right| n^{\prime}\right\rangle\right|^{2}$ is still given by Eq. (23). As a consequence, the polaron correction $\Delta E_{n, l}$ in Eq. (19) becomes

$$
\Delta E_{n, l}=-\sum_{n^{\prime}} \sum_{l^{\prime}} \sum_{j} \sum_{\mathbf{q}_{\|}} \frac{\left|\left\langle l\left|\Gamma_{j}\left(\mathbf{q}_{\|}, z\right)\right| l^{\prime}\right\rangle\right|^{2}\left|\left\langle n\left|e^{i \mathbf{q}_{\|} \cdot \mathbf{r}_{\|}}\right| n^{\prime}\right\rangle\right|^{2}}{\hbar \omega_{\mathbf{q}}-\Delta_{n, l}+\left(n^{\prime}-n\right) \hbar \omega_{c}+E_{l^{\prime}}^{z}-E_{l}^{z}} .
$$

Now, the $\left|\left\langle l\left|\Gamma_{j}\left(\mathbf{q}_{\|}, z\right)\right| l^{\prime}\right\rangle\right|^{2}$ has to be derived for the different phonon modes.

For the interface-phonon modes, we have

$$
\left|\left\langle l\left|\Gamma_{I, \pm}\left(\mathbf{q}_{\|}, z\right)\right| l^{\prime}\right\rangle\right|^{2}=\frac{\sqrt{2} \pi \alpha_{1}\left(1+\gamma_{I} e^{-q_{\|} W}\right)}{A\left(1 / \epsilon_{\infty 1}-1 / \epsilon_{01}\right) q_{\|}} \frac{B_{I, \pm}\left(q_{\|}\right)}{\omega_{I, \pm}\left(q_{\|}\right)}\left|G_{l, l^{\prime}}^{I}\left(q_{\|}\right)\right|^{2}
$$

where

$$
B_{I, \pm}\left(q_{\|}\right)=\frac{\left[\omega_{T 1}^{2}-\omega_{I, \pm}^{2}\left(q_{\|}\right)\right]^{2}\left[\omega_{T 2}^{2}-\omega_{I, \pm}^{2}\left(q_{\|}\right)\right]^{2}}{\epsilon_{1}^{I}\left(\omega_{L 1}^{2}-\omega_{T 1}^{2}\right)\left[\omega_{T 2}^{2}-\omega_{I, \pm}^{2}\left(q_{\|}\right)\right]^{2}+\epsilon_{2}^{I}\left(\omega_{L 2}^{2}-\omega_{T 2}^{2}\right)\left[\omega_{T 1}^{2}-\omega_{I, \pm}^{2}\left(q_{\|}\right)\right]^{2}} .
$$

In the case of the infinite-barrier well model, we obtain

$$
\left|G_{l, l^{\prime}}^{S}\left(q_{\|}\right)\right|^{2}=\left\{\begin{array}{l}
\left(\frac{8 l l^{\prime} \pi^{2} q_{\|} W \tanh \left(q_{\|} W / 2\right)}{\left[\left(q_{\|} W\right)^{2}+\left(l-l^{\prime}\right)^{2} \pi^{2}\right]\left[\left(q_{\|} W\right)^{2}+\left(l+l^{\prime}\right)^{2} \pi^{2}\right]}\right)^{2}, l+l^{\prime}=\text { even } \\
0, \quad l+l^{\prime}=\text { odd },
\end{array}\right.
$$

and

$$
\left|G_{l, l^{\prime}}^{A}\left(q_{\|}\right)\right|^{2}=\left\{\begin{array}{l}
0, \quad l+l^{\prime}=\text { even } \\
\left\{\frac{8 l l^{\prime} \pi^{2} q_{\|} W \operatorname{coth}\left(q_{\|} W / 2\right)}{\left[\left(q_{\|} W\right)^{2}+\left(l-l^{\prime}\right)^{2} \pi^{2}\right]\left[\left(q_{\|} W\right)^{2}+\left(l+l^{\prime}\right)^{2} \pi^{2}\right]}\right]^{2}, \quad l+l^{\prime}=\text { odd } .
\end{array}\right.
$$

For the finite-barrier well model, only the leading term in the sum over $l$ ' will be taken into account,

$$
G_{1,1}^{S}\left(q_{\|}\right)=B_{0}^{2}\left[\frac{2 \cos ^{2}(k W / 2)}{2 k_{1}+q_{\|}}+\left[\frac{1}{q_{\|}}+\frac{q_{\|} \cos (k W)}{4 k^{2}+q_{\|}^{2}}\right] \tanh \left(q_{\|} W / 2\right)+\frac{2 k \sin (k W)}{4 k^{2}+q_{\|}^{2}}\right],
$$

and $G_{1,1}^{A}\left(q_{\|}\right)=0$.

For the slab-phonon modes, we have

$$
\left|\left\langle l\left|\Gamma_{L 1}^{j}\left(\mathbf{q}_{\|}, z\right)\right| l^{\prime}\right\rangle\right|^{2}=\frac{4 \sqrt{2} \pi \alpha_{1}}{A W} \frac{\left|G_{l, l^{\prime}}\right|^{2}}{q_{\|}^{2}+(j \pi / W)^{2}},
$$

where for an infinite-barrier well

$$
\left|G_{l, l^{\prime}}\right|^{2}=\left\{\begin{array}{l}
0, \quad j+l+l^{\prime}=\text { even } \\
{\left[\frac{8 l l^{\prime} j / \pi}{\left[j^{2}-\left(l-l^{\prime}\right)^{2}\right]\left[j^{2}-\left(l+l^{\prime}\right)^{2}\right]}\right]^{2}, j+l+l^{\prime}=\text { odd },}
\end{array}\right.
$$

and, for a finite-barrier well,

$$
G_{1,1}^{j}=\left\{\begin{array}{l}
0, \quad j=\text { even } \\
B_{0}^{2}\left[\frac{W}{j \pi}+\frac{j \pi W \cos (k W)}{(j \pi)^{2}-4(k W)^{2}}\right), j=\text { odd } .
\end{array}\right.
$$

For an infinite-barrier well, the electron wave function is confined in the well, and consequently, the matrix element of an electron interacting with the half-space phonons is zero. For a finite-barrier well, this term is also zero within the leading-term approximation, i.e., $l=l^{\prime}=1$. 
Using Eqs. (33) - (41), we obtain the polaron energy due to electron-interface-phonon and -slab-phonon interaction in such a system. The contribution from interface modes is given by

$$
\Delta E_{n, l}^{I, \pm}=-\frac{\alpha_{1}}{\sqrt{2}\left(1 / \epsilon_{\infty 1}-1 / \epsilon_{01}\right)} \sum_{n^{\prime}=0}^{\infty} \frac{n !}{n^{\prime} !} \sum_{l^{\prime}=1}^{\infty} \int_{0}^{\infty} d q_{\|} \frac{\xi_{\|}^{n^{\prime}-n_{e}} e^{-\xi_{\|}}\left[L_{n}^{n^{\prime}-n_{2}}\left(\xi_{\|}\right)\right]^{2}\left(1+\gamma_{I} e^{-q_{\|} W}\right) B_{I, \pm}\left(q_{\|}\right)\left|G_{l, l^{\prime}}^{I}\left(q_{\|}\right)\right|^{2}}{\omega_{I, \pm}\left(q_{\|}\right)\left[\omega_{I, \pm}\left(q_{\|}\right)-\Delta_{n, l}+\left(n^{\prime}-n\right) \omega_{c}+E_{l}^{\prime z}-E_{l}^{z}\right]} .
$$

The contribution from slab modes is given by

$$
\Delta E_{n, l}^{\text {slab }}=-\frac{2 \sqrt{2} \alpha_{1}}{W} \sum_{n^{\prime}=0}^{\infty} \frac{n !}{n^{\prime} !} \sum_{l^{\prime}=1}^{\infty} \sum_{j=1}^{j_{\max }} \int_{0}^{\infty} d q_{\|} \frac{q_{\|} \xi_{\|}^{n^{\prime}-n} e^{-\xi_{\|}}\left[L_{n}^{n^{\prime}-n}\left(\xi_{\|}\right)\right]^{2}\left|G_{l, l^{\prime}}^{j}\left(q_{\|}\right)\right|^{2}}{\left[q_{\|}^{2}+(j \pi / W)^{2}\right]\left[1-\Delta_{n, l}+\left(n^{\prime}-n\right) \omega_{c}+E_{l}^{\prime z}-E_{l}^{z}\right]} .
$$

The contribution from the half-space phonons is zero.

Note, in Eqs. (42) and (43), $\Delta_{n, l}=\Delta E_{n, l}-\Delta E_{0,1}$, and $\Delta E_{n, l}=\Delta E_{n, l}^{S+}+\Delta E_{n, l}^{S-}+\Delta E_{n, l}^{A+}+\Delta E_{n, l}^{A-}+\Delta E_{n, l}^{\text {slab. For the }}$ lowest Landau level $(n, l)=(0,1)$ we have $\Delta_{0,1}=0$ and it is possible to calculate the polaron energy due to the different phonon modes separately. But for the higher levels $\Delta E_{n, l}$ is calculated self-consistently and Eqs. (42) and (43) have to be combined. Now, we will have the same problem as what we have discussed in the previous section, i.e., the sum over $n^{\prime}$ in Eqs. (42) and (43) converges very slowly in small magnetic field and it results in difficulties to obtain the correct limit results for $\omega_{c}=0$. For the energies below the phonon continuum, $\Delta E_{n, l}$ can be calculated following Eq. (27), and we obtain

$\Delta E_{n, l}=-\sum_{j} \sum_{\mathbf{q}_{\|}} \int_{0}^{\infty} d u e^{-\left(\omega_{\mathbf{q}}-\Delta_{n, l}\right) u} e^{-\mu q_{\|}^{2}} \sum_{n^{\prime}=0}^{n}\left[\begin{array}{l}n \\ n^{\prime}\end{array}\right) \frac{1}{n^{\prime}}\left[\frac{2 \hbar q_{\|}^{2}}{m_{c} \omega_{c}} \sinh ^{2}\left[\frac{\omega_{c} u}{2}\right]\right]^{n^{\prime}} \sum_{l^{\prime}=1}^{\infty}\left|\left\langle l\left|\Gamma_{j}\left(\mathbf{q}_{\|}, z\right)\right| l^{\prime}\right\rangle\right|^{2} e^{-\left(E_{l^{\prime}}^{z}-E_{l}^{z}\right) u}$

With the interface-phonon modes, the above formula reduces to

$$
\begin{aligned}
\Delta E_{n, l}^{I, \pm}=-\frac{\alpha_{1}}{\sqrt{2}\left(1 / \epsilon_{\infty 1}-1 / \epsilon_{01}\right)} \sum_{n^{\prime}=0}^{n}\left[\begin{array}{c}
n \\
n^{\prime}
\end{array}\right] \frac{1}{n^{\prime}} \int_{0}^{\infty} d u \int_{0}^{\infty} d q_{\|} e^{-\left[\omega_{I, \pm}\left(q_{\|}\right)-\Delta_{n, l}-n^{\prime} \omega_{c}\right] u} & \\
& \times \frac{B_{I, \pm}\left(q_{\|}\right)}{\omega_{I, \pm}\left(q_{\|}\right)} \xi_{\|}^{n^{\prime}}\left(1-e^{\left.-\omega_{c} u\right)^{2 n^{\prime}} e^{-\mu q_{\|}^{2}}\left(1+\gamma_{I} e^{-q_{\|} W_{1}}\right.}\right. \\
& \times \sum_{l^{\prime}=1}^{\infty}\left|G_{l, l^{\prime}}^{I}\left(q_{\|}\right)\right|^{2} e^{-\left(E_{l^{\prime}}^{z}-E_{l}^{z}\right) u}
\end{aligned}
$$

where $B_{I, \pm}\left(q_{\|}\right)$is given by Eq. (35), $G_{l, l^{\prime}}^{I}\left(q_{\|}\right)$is given by Eqs. (36), (37), and (38). Equation (45) is valid when $E_{n, l}<E_{0,1}+\hbar \omega_{T 1}$ for the $S-$ mode, $E_{n, l}<E_{0,1}+\hbar \omega_{+}^{\infty}$ for the $S+$ mode, $E_{n, l}<E_{0,1}+\hbar \omega_{-}^{\infty}$ for the $A-$ mode, and $E_{n, l}<E_{0,1}+\hbar \omega_{T 2}$ for the $A+$ mode.

With the slab-phonon modes, Eq. (44) reduces to

$$
\Delta E_{n, l}^{\text {slab }}=-\frac{\sqrt{2} \alpha_{1}}{W} \sum_{n^{\prime}=0}^{n}\left(\begin{array}{c}
n \\
n^{\prime}
\end{array}\right) \frac{1}{n^{\prime}} \int_{0}^{\infty} d u e^{-\left(1-\Delta_{n, l}-n^{\prime} \omega_{c}\right) u}\left(1-e^{-\omega_{c} u}\right)^{n^{\prime}} \sum_{j=1}^{j_{\max }} I_{n^{\prime}}\left(\mu(j \pi / W)^{2}\right) \sum_{l^{\prime}=1}^{\infty}\left|G_{l, l^{\prime}}^{j}\right|^{2} e^{-\left(E_{l^{\prime}}^{z}-E_{l}^{z}\right) u},
$$

where $I_{n}(t)$ is given by Eq. (29). Equation (46) is valid for $E_{n, l}<E_{0,1}+\hbar \omega_{L 1}$. For larger energies Eqs. (42) and (43) have to be used. Now let us consider the case such that $E_{n, l}-E_{0,1}$ is in the region of the interface-phonon energy, i.e.,

$$
\begin{aligned}
& \hbar \omega_{-}^{\infty} \geq E_{n, l}-E_{0,1} \geq \hbar \omega_{T 1} \text { for } S-\text { mode }, \\
& \hbar \omega_{L 2} \geq E_{n, l}-E_{0,1} \geq \hbar \omega_{+}^{\infty} \text { for } S+\text { mode }, \\
& \hbar \omega_{L 1} \geq E_{n, l}-E_{0,1} \geq \hbar \omega_{-}^{\infty} \text { for } A-\text { mode }, \\
& \hbar \omega_{+}^{\infty} \geq E_{n, l}-E_{0,1} \geq \hbar \omega_{T 2} \text { for } A+\text { mode } .
\end{aligned}
$$

In the above energy region, the denominator in Eq. (42) $\left(n^{\prime}=0\right.$ term) can become zero because of the magnetophonon resonance (for $n \geq 1$ ). This term can be calculated in the following way:

$$
\Delta E\left(n^{\prime}=0\right)=\frac{1}{n !} \sum_{l^{\prime}=1}^{\infty} \int_{\omega_{1}}^{\omega_{2}} d \omega \frac{f(\omega)}{\omega-\omega_{0}}
$$

with 


$$
f(\omega)=\frac{4}{W} \frac{\left(\omega_{T 1}^{2}-\omega^{2}\right)^{2}\left(\omega_{T 2}^{2}-\omega^{2}\right)^{2} \xi_{\|}^{n} e^{-\xi_{\|}}\left|G_{l, l^{\prime}}\left(q_{\|}\right)\right|^{2}}{\left[\epsilon_{\infty 2}\left(\omega_{L 2}^{2}-\omega^{2}\right)\left(\omega_{T 1}^{2}-\omega^{2}\right)\right]^{2}-\left[\epsilon_{\infty 1}\left(\omega_{L 1}^{2}-\omega^{2}\right)\left(\omega_{T 2}^{2}-\omega^{2}\right)\right]^{2}}
$$

and

$$
q_{\|}=\frac{1}{W} \ln \left(\frac{\gamma_{I}\left[\epsilon_{\infty 1}\left(\omega_{L 1}^{2}-\omega^{2}\right)\left(\omega_{T 2}^{2}-\omega^{2}\right)-\epsilon_{\infty 2}\left(\omega_{L 2}^{2}-\omega^{2}\right)\left(\omega_{T 1}^{2}-\omega^{2}\right)\right]}{\epsilon_{\infty 1}\left(\omega_{L 1}^{2}-\omega^{2}\right)\left(\omega_{T 2}^{2}-\omega^{2}\right)+\epsilon_{\infty 2}\left(\omega_{L 2}^{2}-\omega^{2}\right)\left(\omega_{T 1}^{2}-\omega^{2}\right)}\right],
$$

where $\hbar \omega_{0}=\Delta_{n, l}+n \hbar \omega_{c}+E_{l^{\prime}}^{z}-E_{l}^{z}$, and the integration interval in Eq. (47a) is given by

$$
\left(\omega_{1}, \omega_{2}\right)= \begin{cases}\left(\omega_{T 1}, \omega_{-}^{\infty}\right) & \text { for } S^{-} \text {mode } \\ \left(\omega_{L 2}, \omega_{+}^{\infty}\right) & \text { for } S^{+} \text {mode } \\ \left(\omega_{L 1}, \omega_{-}^{\infty}\right) & \text { for } A^{-} \text {mode } \\ \left(\omega_{T 2}, \omega_{+}^{\infty}\right) & \text { for } A^{+} \text {mode } .\end{cases}
$$

Now, the energy shift of the Landau levels due to the interface and slab phonons can be obtained by solving the following nonlinear equation:

$$
\Delta E_{n, l}=\operatorname{Re} F\left(\Delta E_{n, l}\right)
$$

with

$$
F\left(\Delta E_{n, l}\right)=\sum_{I, \pm} \Delta E_{n, l}^{I, \pm}+\Delta E_{n, l}^{\mathrm{slab}},
$$

where $\Delta E_{n, l}^{I, \pm}$ is given by Eqs. (42), (45), and (47), and $\Delta E_{n, l}^{\text {slab }}$ is given by Eqs. (43) and (46).

The polaron binding energy of the lowest two Landau levels in different magnetic fields $\left(\omega_{c} / \omega_{\mathrm{LO}}=0,0.5\right.$, and $1.0)$ is plotted as a function of the well width in Figs. 3(a) and $3(\mathrm{~b})$ for the infinite-barrier well. The dashed curve gives the correction induced by $3 \mathrm{D}$ phonons and the solid curve respects the result with interface and slab phonons. The calculation shows that (1) the polaron correction increases (a) with increasing magnetic field and (b) with decreasing well width; (2) in the case of $3 \mathrm{D}$ phonon modes, it starts from the ideal-2D (I2D) value of GaAs at $W=0$ and decreases monotonously to the 3D value of GaAs in the limit $W \rightarrow \infty$. While for the result with interface- and slab-phonon modes it starts from the I2D value of AlAs at $W=0$ and decreases to the $3 \mathrm{D}$ value of $\mathrm{GaAs}$ when $W \rightarrow \infty$; (3) the binding energy with interface and slab phonons is always larger as compared to the 3D phonon; and (4) in the limit of zero magnetic field, i.e., $\omega_{c}=0$, the correction to the two lowest Landau levels, i.e., $\Delta E_{0,1}$ and $\Delta E_{1,1}$, are the same as it should be.

For a finite-barrier well, the polaron binding energy of the lowest two Landau levels is given in Figs. 4(a) and 4(b) as a function of the well width. In this case we were forced to make the leading-term approximation for the electric energy levels as is also done in all previous papers on this problem. We expect that when the separation between the electric levels becomes smaller than the LOphonon energy $\hbar \omega_{\text {LO }}$, this approximation breaks down. One of the consequences of this approximation is that the polaron correction goes to zero for $W \rightarrow 0$ and $W \rightarrow \infty$.
There exists a well width $W \simeq 6 \AA$ where $\left|\Delta E_{n, l}\right|$ is maximum. The position of this turns out to be almost not influenced by (1) the magnetic field, and (2) whether or not we consider 3D phonons or the case of interface and slab modes. On the large well-width side we have $E_{2}^{z}-E_{1}^{z} \simeq \hbar \omega_{\text {LO }}$ for $W \simeq 285 \AA$ and the results are only indicative in the region $W>285 \AA$, while on the low well-width side this is the case for $W<6 \AA$.

Comparing the results of infinite- and finite-barrier quantum wells, we find that, at the same magnetic field and well width, the polaron correction energy in an infinite-barrier well is always larger than that in a finitebarrier well. For wide and very narrow wells this is mainly a result from the leading-term approximation. In the other cases it is a consequence of the fact that the electron wave function is more extended in a finitebarrier well.

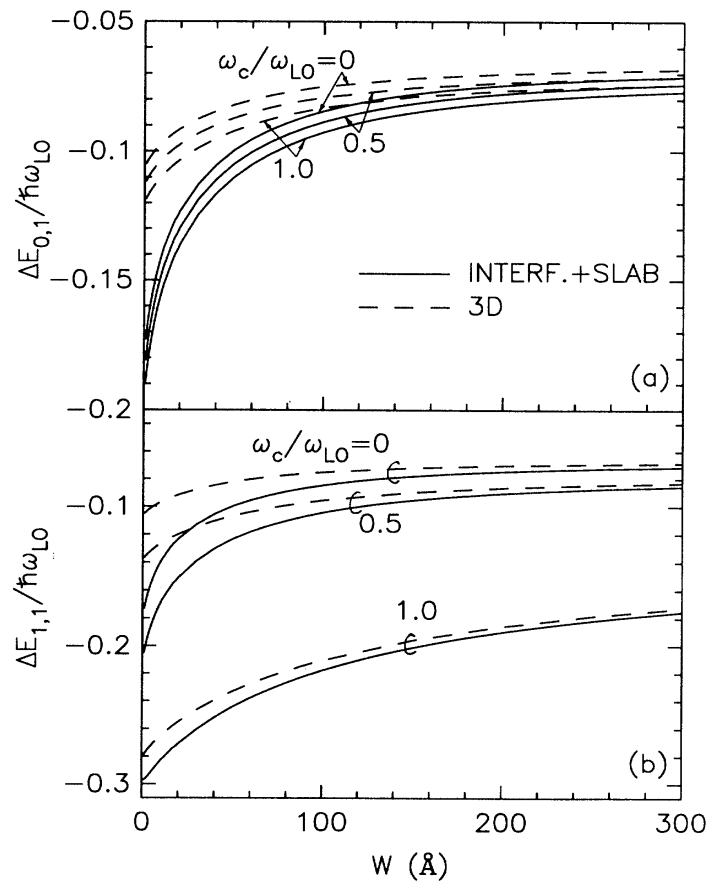

FIG. 3. The polaron binding energy of the (a) $n=0$ and (b) $n=1$ Landau levels as a function of the width of the infinitebarrier quantum well in different magnetic fields $\omega_{c} / \omega_{\mathrm{LO}}=0$, 0.5 , and 1.0, with interface and slab phonons (solid curves) and 3D LO phonons (dashed curves). 


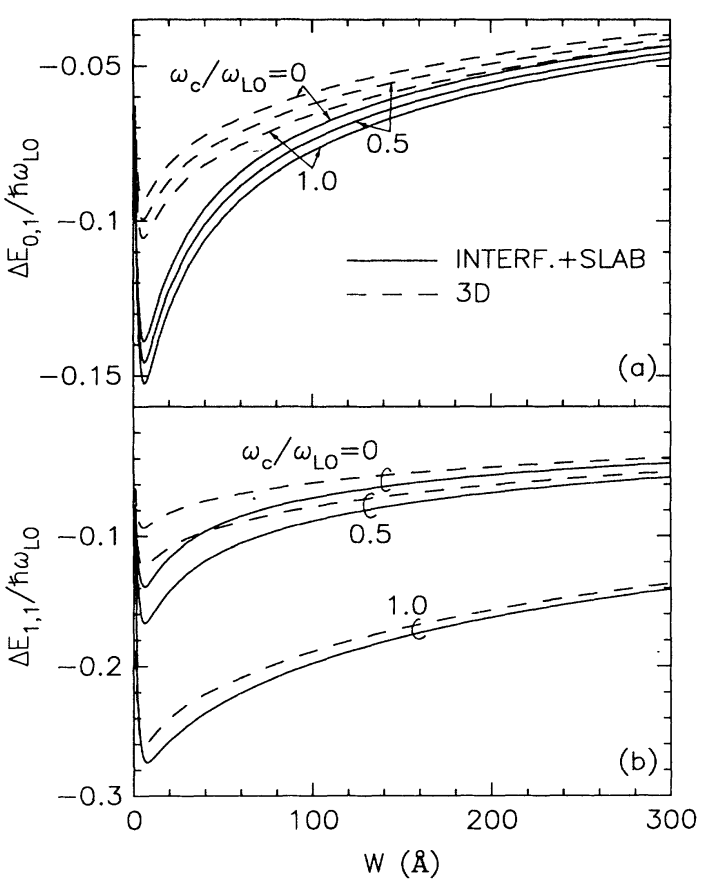

FIG. 4. The polaron binding energy of (a) $n=0$ and (b) $n=1$ Landau levels as a function of the width of a finite-barrier quantum well in different magnetic fields $\omega_{c} / \omega_{\mathrm{LO}}=0,0.5$, and 1.0, with interface and slab phonons (solid curves) and 3D LO phonons (dashed curves).

In a cyclotron-resonance experiment, the cyclotronresonance frequency $\omega^{*}$ refers to the transition between $E_{0,1}$ and $E_{1,1}$, namely $\hbar \omega^{*}=E_{1,1}-E_{0,1}$. And consequently, the cyclotron-resonance mass $m^{*}$ is defined by

$$
\frac{m^{*}}{m_{c}}=\frac{\omega_{c}}{\omega^{*}}=\frac{\omega_{c}}{\omega_{c}+\left(\Delta E_{1,1}-\Delta E_{0,1}\right) / \hbar} .
$$

The dependence of the cyclotron mass $\left(m^{*}>m_{c}\right)$ on the well width is given in Figs. 5(a) for an infinite-barrier well and $5(\mathrm{~b})$ for a finite-barrier well in a magnetic field such that $\omega_{c} / \omega_{\mathrm{LO}}=0,0.5$, and 1.0. The dashed curves respect the cyclotron mass with $3 \mathrm{D}$ phonons, and the solid curves give the results with interface and slab phonons. Figure 5(a) shows that, with 3D phonons, the polaron cyclotron mass in an infinitely high barrier well starts from the I2D mass of GaAs at $W=0$ and decreases to the $3 \mathrm{D}$ value for $W \rightarrow \infty$. In the small magnetic-field limit, the cyclotron mass with interface and slab phonons is larger than the result with $3 \mathrm{D}$ phonons, which is consistent with what we got in the absence of the magnetic field. ${ }^{44}$ The interesting thing is that, in the highmagnetic-field case $\omega_{c} / \omega_{\mathrm{LO}}=0.5$ and 1.0 , the cyclotron mass with interface and slab phonons becomes smaller than that with $3 \mathrm{D}$ LO phonons, especially in a narrow well. At $\omega_{c} / \omega_{\mathrm{LO}}=1.0$, a peak in $m^{*}$ vs $\omega_{c}$ occurs at $W=28 \AA$. To understand this, let us consider the limit case such that the well width goes to zero for the present model. In such a situation, with interface-phonon modes, the I2D system of AlAs should be reached. As a conse-

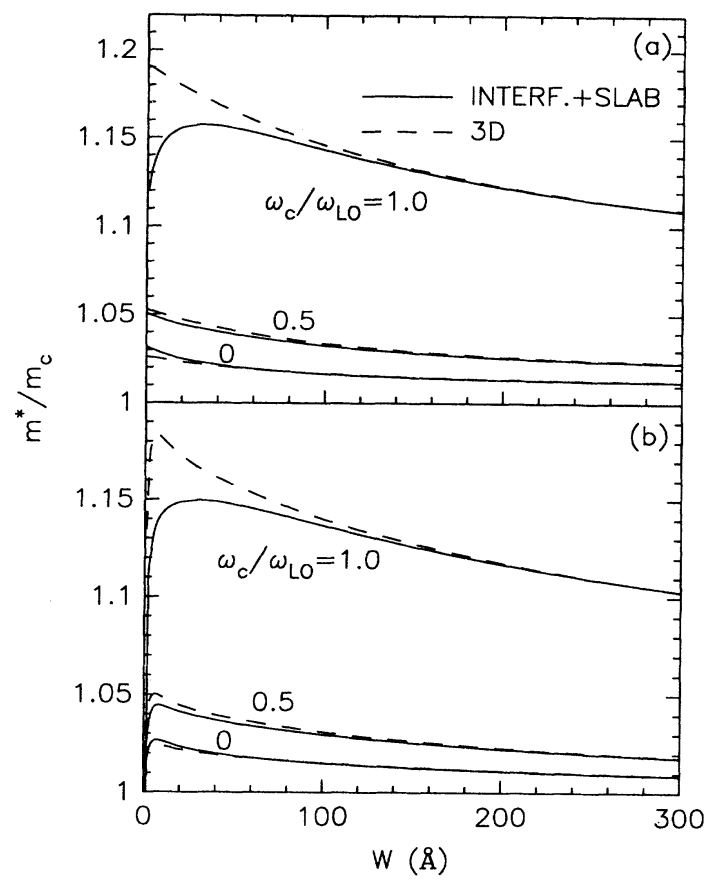

FIG. 5. The polaron cyclotron mass as a function of the well width for (a) an infinite-barrier quantum well and (b) for a finite-barrier quantum well in different magnetic fields $\omega_{c} / \omega_{\mathrm{LO}}=0,0.5$, and 1.0 , with interface and slab phonons (solid curves) and 3D LO phonons (dashed curves).
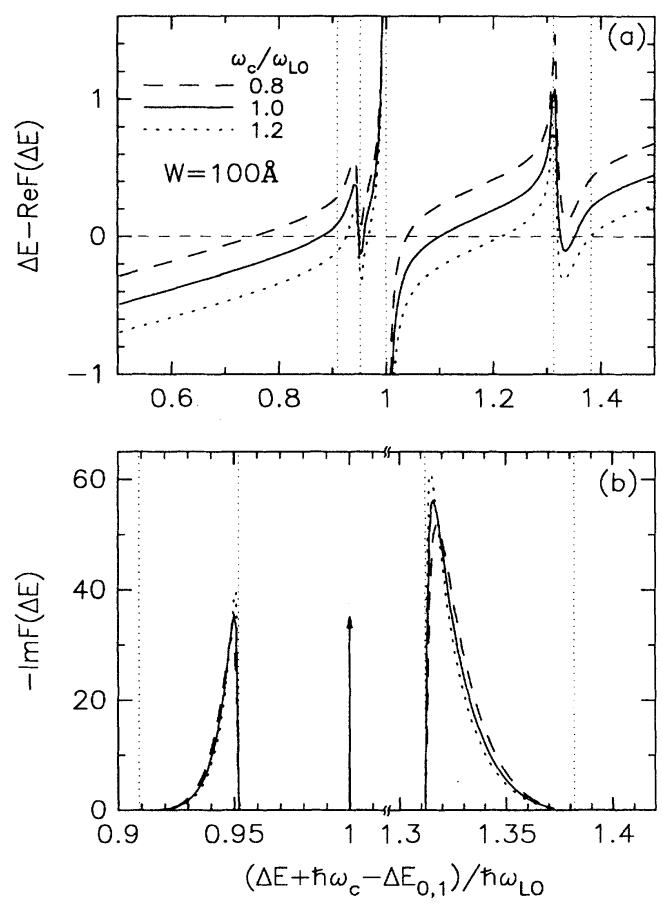

FIG. 6. (a) $\Delta E-\operatorname{Re} F(\Delta E)$ and (b) $\operatorname{Im}(\Delta E)$ as a function of $\Delta E+\hbar \omega_{c}-\Delta E_{0,1}$, for a 100 - $\AA$ quantum well with interface and slab phonons. The vertical thin dotted lines indicate the energies $\hbar \omega_{T 1}, \hbar \omega_{-}^{\infty}, \hbar \omega_{L 1}, \hbar \omega_{+}^{\infty}$, and $\hbar \omega_{L 2}$. The upward arrow in (b) indicates a $\delta$ function. The magnetic fields are such that $\omega_{c} / \omega_{\mathrm{LO}}=0.8,1.0$, and 1.2 . 
quence, the result of the I2D AlAs system is obtained. For GaAs the magnetophonon resonance occurs at $\omega_{c}=\omega_{\mathrm{LO}}$, and, at this moment, it is responsible for a large polaron correction. But the LO-phonon energy of AlAs is $\omega_{L 2}=1.38 \omega_{\mathrm{LO}}$, the resonance happens at higher magnetic field. Therefore, for a narrow well the polaron cyclotron mass with 3D phonon modes is larger than that with interface- and slab-phonon modes around $\omega_{c}=\omega_{\mathrm{LO}}$.

For a finite-barrier well, the result is given in Fig. 5(b). The cyclotron mass in this case is smaller than the corresponding result in Fig. 5(a). While for $W \rightarrow 0$ and
$W \rightarrow \infty$ the cyclotron mass goes to zero because of the leading-term approximation. Also, there is a peak in $m^{*}$ vs $W$ at $W \simeq 8 \AA$ except for the top solid curve. The peak in the top solid curve (with interface and slab phonons, $\left.\omega_{c} / \omega_{\mathrm{LO}}=1.0\right)$, occurs around $W=28 \AA$ which is mainly induced by the interface-phonon modes.

When the interface- and slab-phonon modes are considered, the calculation and the results become more complicated. To make the problem transparent, we have calculated the real and imaginary part of $F(\Delta E)$ in Eq. (49) as a function of $\Delta E$ in different magnetic fields. In
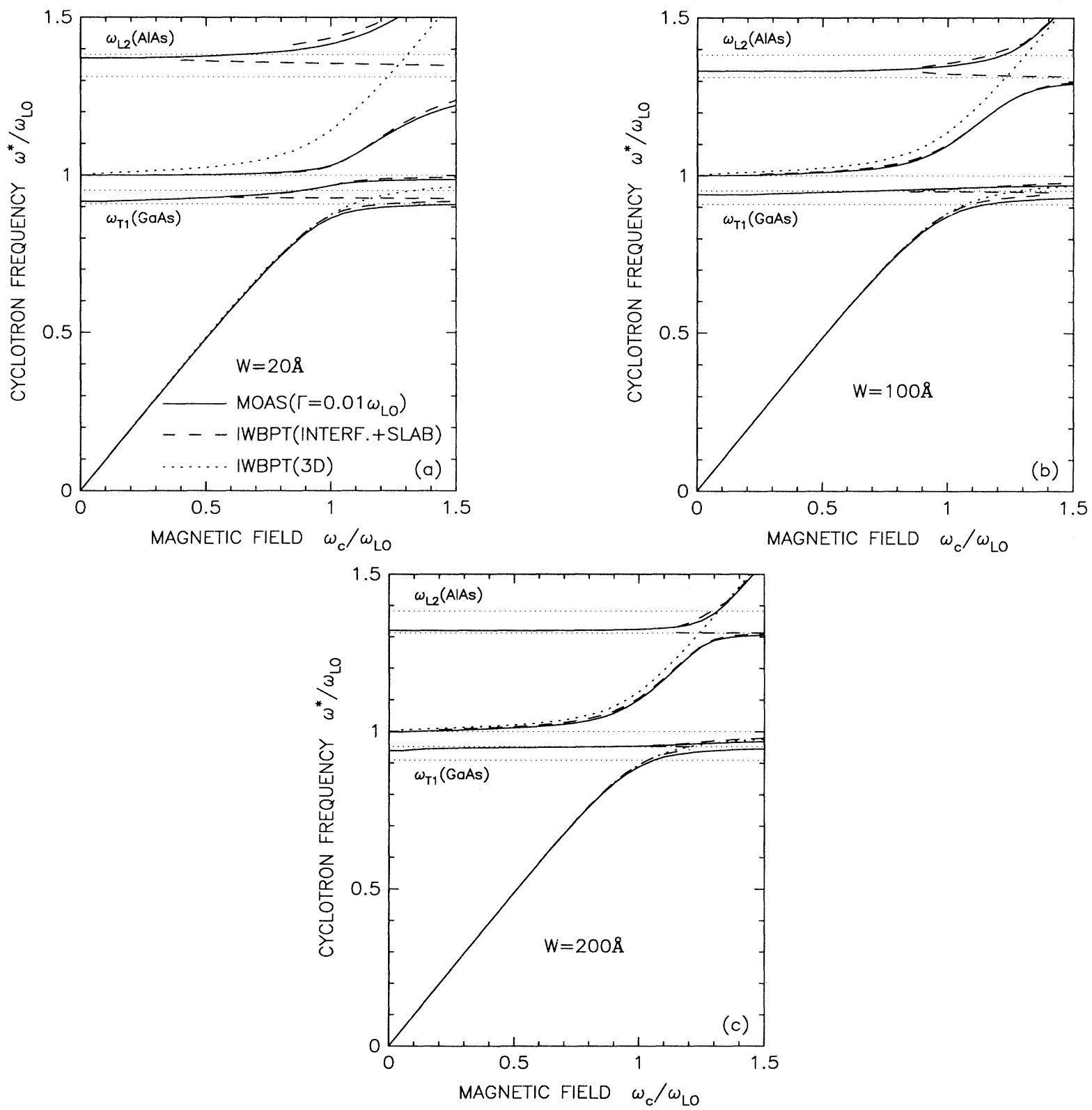

FIG. 7. The cyclotron frequency as a function of magnetic field in (a) 20-, (b) 100-, and (c) 200- $\AA$ GaAs/AlAs quantum wells. The solid curves represent the position of the four peaks in the magneto-optical absorption spectrum with interface and slab phonons $\left(\Gamma / \hbar \omega_{\text {LO }}=0.01\right)$. The dashed and dotted curves are from the IWBPT results with interface and slab phonons and only $3 D$ LOphonon modes, respectively. 
Figs. 6(a) $\Delta E-\operatorname{Re} F(\Delta E)$ and 6(b) $\operatorname{Im} F(\Delta E)$ are plotted as a function of $\Delta E+\hbar \omega_{c}-\Delta E_{0,1}$ for the level $(n, l)=(1,1)$ in different magnetic fields with a finitebarrier well model, $W=100 \AA$. The polaron correction $\Delta E_{1,1}$ is determined by the equation $\Delta E-\operatorname{Re} F(\Delta E)=0$. It is seen that $\operatorname{Re} F(\Delta E)$ is discontinuous at $\hbar \omega_{c}+\Delta E-\Delta E_{0,1}=E_{1,1}-E_{0,1}=\hbar \omega_{\mathrm{LO}}$. This is due to the onset of slab-mode phonon scattering. The $\operatorname{Im} F(\Delta E)$ gives a $\delta$ function at this point. For a finite-barrier well, only the symmetric interface-phonon and slab-phonon modes contribute to the polaron effect within the leading-term approximation. Around the symmetric interface-phonon energy magnetointerface-opticalphonon resonances occur. But the real part of $F(\Delta E)$ is still continuous in the region of the interface-phonon energies due to the dispersiveness of the interface-phonon modes. The imaginary part of $F(\Delta E)$ is now different from zero. From Fig. 6(a) we observe that Eq. (49a) can have two roots in the low-magnetic-field region: one $E_{1,1}-E_{0,1}$ below the interface-phonon energy of the $S-$ modes, another above the LO-phonon energy. But in large magnetic fields, Eq. (49a) may have up to six roots.

The transition energy $\hbar \omega^{*}=E_{1,1}-E_{0,1}$, as obtained from the roots of Eq. (49), is plotted (dashed curves) as a function of magnetic field in Fig. 7 for different well widths (a) $W=20 \AA$, (b) $W=100 \AA$, and (c) $W=200 \AA$. The dotted curves are the results when only bulk LOphonon modes of GaAs were included. The solid curves are the peak positions in the magneto-optical absorption spectrum which will be discussed later. The cyclotron frequency, when including interface and slab phonons, deviates appreciably from the result with only the bulk LO phonons when $\omega^{*} \geq \omega_{\text {TO }}(\mathrm{GaAs})$. With $3 \mathrm{D}$ phonons, the frequency $\omega^{*}$ splits into two branches around $\omega_{\mathrm{LO}}$, and the lower branch is pinned at $\omega_{\mathrm{LO}}$ in high magnetic field. With the interface and slab phonons, however, the cyclotron-resonance spectrum not only splits at $\omega_{\text {LO }}$, but also around the $q_{\|} \rightarrow \infty, S-$, and $S+$ mode frequencies. The dashed curves in Fig. 7 can be divided into six sections. Four of them increase monotonously as the magnetic field increases, and the other two-one occurs in the energy region of the $S$ - mode and the other in that of the $S+$ mode - are nearly flat.

A direct measure for the strength of the electronphonon interaction is the splitting $\Delta$ of the Landau level at $\omega_{c}=\omega_{\mathrm{LO}}$ around $E_{0,1}+\hbar \omega_{\mathrm{LO}}$. This splitting is shown in Fig. 8 as a function of the well width for 3D phonons (dashed curves) and for the case of interface and slab

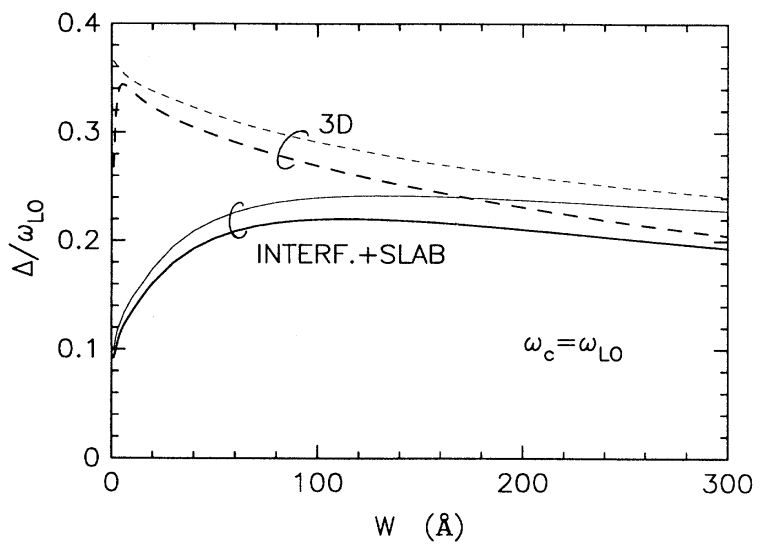

FIG. 8. The splitting of the $n=1$ Landau level at $\omega_{c}=\omega_{\text {LO }}$ as a function of the well width with $3 \mathrm{D} \mathrm{GaAs}$ LO-phonon modes (dashed curves) and with interface- and slab-phonon modes (solid curves). The thick and thin curves present the results for the finite- and infinite-barrier wells, respectively.

modes (solid curves). The influence of the confinement potential is shown for the infinite-barrier (thin curves) and finite-barrier (thick curves) case.

\section{MAGNETO-OPTICAL ABSORPTION SPECTRUM}

In this section we will calculate the magneto-optical absorption spectrum as induced by the interaction with interface- and slab-phonon modes following the method in Refs. 50, 51, and 19. Within the linear-response theory the frequency-dependent magneto-optical absorption spectrum is proportional to

$$
\frac{-\operatorname{Im} \Sigma(\omega)}{\left[\omega-\omega_{c}-\operatorname{Re} \Sigma(\omega)\right]^{2}+[\operatorname{Im} \Sigma(\omega)]^{2}},
$$

where $\Sigma(\omega)$ is the so-called memory function. We obtain the memory function with interface- and slab-phonon modes

$$
\Sigma(\omega)=\frac{1}{\omega} \int_{0}^{\infty} d t\left(1-e^{i \omega t}\right) \operatorname{Im} F(t),
$$

where

$$
\begin{aligned}
F(t)=-\sum_{j} \sum_{\mathbf{q}_{\|}} \frac{q_{\|}^{2}}{\hbar m_{c}}\{ & {\left[1+n\left(\omega_{j}\left(q_{\|}\right)\right)\right]\left\langle\Gamma_{j}\left(\mathbf{q}_{\|}, z(t)\right) \Gamma_{j}^{*}\left(\mathbf{q}_{\|}, z(0)\right)\right\rangle\left\langle e^{i \mathbf{q}_{\|} \cdot \mathbf{r}_{\|}(t)} e^{-i \mathbf{q}_{\|} \cdot \mathbf{r}_{\|}(0)}\right\rangle } \\
& \left.-n\left(\omega_{j}\left(q_{\|}\right)\right)\left\langle\Gamma_{j}^{*}\left(\mathbf{q}_{\|}, z(0)\right) \Gamma_{j}\left(\mathbf{q}_{\|}, z(t)\right)\right\rangle\left\langle e^{-i \mathbf{q}_{\|} \cdot \mathbf{r}_{\|}(0)} e^{i \mathbf{q}_{\|} \cdot \mathbf{r}_{\|}(t)}\right\rangle\right\} e^{-i \omega_{j}\left(q_{\|}\right) t},
\end{aligned}
$$


where $n\left(\omega_{j}\left(q_{\|}\right)\right)=\left(e^{\beta \hbar \omega_{j}\left(q_{\|}\right)}-1\right)^{-1}$ is the number of the phonons in the $j$ th mode with energy $\hbar \omega_{j}\left(q_{\|}\right)$, and $\left\langle\Gamma_{j}\left(\mathbf{q}_{\|}, z(t)\right) \Gamma_{j}^{*}\left(\mathbf{q}_{\|}, z(0)\right)\right\rangle$ and $\left\langle e^{i \mathbf{q}_{\|} \cdot \mathbf{r}_{\|}(t)} e^{-i \mathbf{q}_{\|} \cdot \mathbf{r}_{\|}(0)}\right\rangle$ are correlation functions. In the following, we will concentrate on the zero-temperature case and calculate the correlation function to zero order in the electron-phonon interaction.

At zero temperature, $n\left(\omega_{j}\left(q_{\|}\right)\right)=0$, and the densitydensity correlation function in the $x y$ plane reduces to

$$
\begin{aligned}
\left\langle e^{i \mathbf{q}_{\|} \cdot \mathbf{r}_{\|}(t)} e^{-i \mathbf{q}_{\|} \cdot \mathbf{r}_{\|}(0)}\right\rangle & =\left\langle n=0\left|e^{i \mathbf{q}_{\|} \cdot \mathbf{r}_{\|}(t)} e^{-i \mathbf{q}_{\|} \cdot \mathbf{r}_{\|}(0)}\right| n=0\right\rangle \\
& =e^{-\Gamma^{2} t^{2} / 4} \exp \left[-\xi_{\|}\left(1-e^{-i \omega_{c} t}\right)\right],
\end{aligned}
$$

where $|n=0\rangle$ indicates the lowest unperturbed Landau level, and $\Gamma$ is the Landau-level broadening parameter. For convenience this parameter is taken independent of the Landau-level number. The correlation function $\left\langle\Gamma_{j}\left(\mathbf{q}_{\|}, z(t)\right) \Gamma_{j}^{*}\left(\mathbf{q}_{\|}, z(0)\right)\right\rangle$ can be calculated in the following way:

$$
\begin{aligned}
& \left\langle\Gamma_{j}\left(\mathbf{q}_{\|}, z(t)\right) \Gamma_{j}^{*}\left(\mathbf{q}_{\|}, z(0)\right)\right\rangle \\
& \quad=\left\langle l=1\left|\Gamma_{j}\left(\mathbf{q}_{\|}, z(t)\right) \Gamma_{j}^{*}\left(\mathbf{q}_{\|}, z(0)\right)\right| l=1\right\rangle \\
& \quad=\sum_{l^{\prime}=1}^{\infty}\left|\left\langle 1\left|\Gamma_{j}\left(\mathbf{q}_{\|}, z\right)\right| l^{\prime}\right\rangle\right|^{2} e^{-i\left(E_{l^{\prime}}^{z}-E_{1}^{z}\right) t},
\end{aligned}
$$

where $|l=1\rangle$ indicates the electron state in the lowest electric subband. For the finite-barrier well case only the leading term in the sum over $l^{\prime}$ will be included and $F(t)$ reduces to

$$
F(t)=-\sum_{j} \sum_{\mathbf{q}_{\|}} \frac{q_{\|}^{2}}{\hbar m_{c}}\left|\left\langle 1\left|\Gamma_{j}\left(\mathbf{q}_{\|}, z\right)\right| 1\right\rangle\right|^{2} \exp \left(-\frac{\Gamma^{2} t^{2}}{4}-\xi_{\|}\left(1-e^{-i \omega_{c} t}\right)-i \omega_{j}\left(q_{\|}\right) t\right) .
$$

For the interface-phonon modes,

$$
\left.F^{I, \pm}(t)=-\frac{\alpha_{1}}{\sqrt{2}\left(1 / \epsilon_{\infty 1}-1 / \epsilon_{01}\right)} \int_{0}^{\infty} d q_{\|} \frac{q_{\|}^{2}\left(1+\gamma_{I} e^{-q_{\|} w}\right)}{\omega_{I, \pm}\left(q_{\|}\right)}\left|G_{l, l^{\prime}}^{I}\left(q_{\mid}\right)\right|^{2} \exp \mid-\frac{\Gamma^{2} t^{2}}{4}-\xi_{\|}\left(1-e^{-i \omega_{c} t}\right)-i \omega_{I, \pm}\left(q_{\|}\right) t\right),
$$

and for the slab-phonon modes

$$
F^{\text {slab }}(t)=-\frac{2 \sqrt{2} \alpha_{1}}{W} \sum_{j} \int_{0}^{\infty} d q_{\|} \frac{q_{\|}^{3}\left|G_{l, l^{\prime}}\right|^{2}}{q_{\|}^{2}+(j \pi / W)^{2}} \exp \left(-\frac{\Gamma^{2} t^{2}}{4}-\xi_{\|}\left(1-e^{-i \omega_{c} t}\right)-i \omega_{\mathrm{LO}} t\right) .
$$

Then $F(t)$ is given by the sum $F(t)=F^{S,+}(t)+F^{S,-}(t)+F^{\text {slab }}(t)$.

At last, we obtain the memory function with the interface and slab phonons, at zero temperature,

$$
\begin{aligned}
\operatorname{Re} \Sigma(\omega)= & -\frac{\alpha_{1}}{\sqrt{2}\left(1 / \epsilon_{\infty 1}-1 / \epsilon_{01}\right)} \sum_{I, \pm} \int_{0}^{\infty} d q_{\|} \frac{q_{\|}^{2}\left(1+\gamma_{I} e^{-q_{\|} W}\right)}{\omega_{I, \pm}\left(q_{\|}\right)} B_{I, \pm}\left(q_{\|}\right)\left|G_{l, l^{\prime}}^{I}\left(q_{\mid}\right)\right|^{2} \\
& \times \sum_{n=0}^{\infty} \frac{\xi_{\|}^{n} e^{-\xi_{\|}}}{n ! \omega \Gamma}\left[2 D\left(\frac{\varepsilon_{n}^{I}}{\Gamma}\right)-D\left(\frac{\varepsilon_{n}^{I}+\omega}{\Gamma}\right)-D\left(\frac{\varepsilon_{n}^{I}-\omega}{\Gamma}\right)\right] \\
& +\frac{2 \sqrt{2} \alpha_{1}}{W} \sum_{j=1}^{j_{\max }} \int_{0}^{\infty} d q_{\|} \frac{q_{\|}^{3}\left|G_{l, l^{\prime}}\right|^{2}}{q_{\|}^{2}+(j \pi / W)^{2}} \sum_{n=0}^{\infty} \frac{\xi_{\|}^{n} e^{-\xi_{\|}}}{n ! \omega \Gamma}\left[2 D\left(\frac{\varepsilon_{n}^{\mathrm{LO}}}{\Gamma}\right)-D\left(\frac{\varepsilon_{n}^{\mathrm{LO}}+\omega}{\Gamma}\right)-D\left(\frac{\varepsilon_{n}^{\mathrm{LO}}-\omega}{\Gamma}\right)\right],
\end{aligned}
$$

and

$$
\begin{aligned}
\operatorname{Im} \Sigma(\omega)= & -\frac{\alpha_{1}}{\sqrt{2}\left(1 / \epsilon_{\infty 1}-1 / \epsilon_{01}\right)} \sum_{I, \pm} \int_{0}^{\infty} d q_{\|} \frac{q_{\|}^{2}\left(1+\gamma_{I} e^{-q_{\|} W}\right)}{\omega_{I, \pm}\left(q_{\|}\right)} B_{I, \pm}\left(q_{\|}\right)\left|G_{l, l^{\prime}}^{I}\left(q_{\mid}\right)\right|^{2} \\
& \times \sum_{n=0}^{\infty} \frac{\sqrt{\pi} \xi_{\|}^{n} e^{-\xi_{\|}}}{2 n ! \omega \Gamma}\left\{\exp \left[-\left(\frac{\varepsilon_{n}^{I}-\omega}{\Gamma}\right]^{2}\right]-\exp \left[-\left[\frac{\varepsilon_{n}^{I}+\omega}{\Gamma}\right]^{2}\right]\right\} \\
& +\frac{2 \sqrt{2} \alpha_{1}}{W} \sum_{j=1}^{j} \int_{0}^{\infty} d q_{\|} \frac{q_{\|}^{3}\left|G_{l, l^{\prime}}\right|^{2}}{q_{\|}^{2}+(j \pi / W)^{2}} \sum_{n=0}^{\infty} \frac{\sqrt{\pi} \xi_{\|}^{n} e^{-\xi_{\|}}}{2 n ! \omega \Gamma}\left\{\exp \left[-\left[\frac{\varepsilon_{n}^{\mathrm{LO}}-\omega}{\Gamma}\right]^{2}\right]-\exp \left[-\left(\frac{\varepsilon_{n}^{\mathrm{LO}}+\omega}{\Gamma}\right]^{2}\right]\right\},
\end{aligned}
$$


where $\varepsilon_{n}^{I}=n \omega_{c}+\omega_{I, \pm}\left(q_{\|}\right), \varepsilon_{n}^{\mathrm{LO}}=n \omega_{c}+\omega_{\mathrm{LO}}$, and $D(x)$ is the Dawson integral.

We have calculated numerically the real and imaginary parts of the memory function, including interface- and slab-phonon modes in a finite-barrier GaAs/AlAs quantum well from which we obtained the magneto-optical absorption spectrum. The cyclotron-resonance frequency $\omega^{*}$ is determined from the peak position in the absorption spectrum. When $\operatorname{Im} \Sigma(\omega)=0$ the absorption peak is a $\delta$ function whose position is given by the nonlinear equation

$$
\omega-\omega_{c}-\operatorname{Re} \Sigma(\omega)=0 .
$$

For a $100-\AA$ GaAs/AlAs quantum well, the function $\omega-\omega_{c}-\operatorname{Re} \Sigma(\omega)$ and the imaginary part of the memory function $\operatorname{Im} \Sigma(\omega)$ are plotted in Figs. 9(a) and 9(b), respectively, at different magnetic fields $\omega_{c} / \omega_{\text {LO }}=0.8,1.0$, and 1.2 with a Landau-level broadening parameter $\Gamma=0.01 \omega_{\text {LO }}$. The vertical thin-dashed lines indicate the frequencies $\omega_{T 1}, \omega_{-}^{\infty}, \omega_{L 1}, \omega_{+}^{\infty}$, and $\omega_{L 2}$. It is seen that the $\operatorname{Im} \Sigma(\omega)$ has a peak around the phonon frequencies and when the frequency $\omega$ deviates from the phonon frequencies the imaginary part of the memory function tends towards zero as shown in Fig. 9(b) for $\Gamma=0.01 \omega_{\mathrm{LO}}$. In such a case, the peak position in the magneto-optical absorption spectrum, which is given by Eq. (51), is determined by Eq. (61) and there exists a $\delta$-function absorption at $\omega^{*}$. Actually, Fig. 9(a) is similar to Fig. 6(a). Because the Landau-level broadening was included in the memory function, $\operatorname{Re} \Sigma(\omega)$ is continuous at $\omega=\omega_{\mathrm{LO}}$. For $\Gamma \neq 0$ we determined the cyclotron frequency $\omega^{*}$ from the peak position in the absorption spectrum and not from the equation $\omega-\omega_{c}-\operatorname{Re} \Sigma(\omega)=0$ directly.

Figure 9(c) gives the corresponding magneto-optical absorption spectrum. The upward arrows indicate a $\delta$ function absorption. With interface and slab phonons, the spectrum structure is quite different from that with 3D LO-phonon modes and is more complex. It is apparent that there are four distinct absorption peaks in the spectrum. The position of the first peak starts from zero at $\omega_{c}=0$ and is pinned below $\omega_{S,-}\left(q_{\|} \rightarrow \infty\right)$ in the highmagnetic-field limit. The second peak occurs for $\omega_{\mathrm{LO}}>\omega^{*} \geq \omega_{S-}$, the third peak for $\omega_{S+}>\omega^{*} \geq \omega_{\mathrm{LO}}$, and the fourth peak for $\omega^{*} \geq \omega_{S+}$. It is noticeable that in high magnetic fields, the first peak occurs for $\omega^{*}>\omega_{T 1}$, which is in the reststrahlen region of GaAs. It is also found that the fourth peak may occur in the reststrahlen region of AlAs.

The frequency $\omega^{*}$ from the four peaks in the absorption spectrum are given by the solid curves in Fig. 7. With interface- and slab-phonon modes the cyclotron frequency splits into four branches, which is similar to the result from IWBPT. Actually, there exist three other very weak peaks in the absorption spectrum. Two of them are responsible for the nearly flat dashed line (IWBPT result) in the range of the $S-$ and $S+$ interface-phonon frequencies, and another from the slab phonons. These three peaks are due to the phonon emission and have almost no weight in the absorption spectrum. Therefore they will not be considered in the present study.

The oscillator strength of the different absorption peaks was calculated and is given in Figs. 10(a) for $W=20 \AA$, (b) for $W=100 \AA$, and (c) for $W=200 \AA$. They are indicated by $\omega_{1}^{*}, \omega_{2}^{*}, \omega_{3}^{*}$, and $\omega_{4}^{*}$ for the four peaks and are in increasing order of frequency. The oscillator strengths of the absorption spectrum with only 3D bulk LO phonons of GaAs are given by the thin-solid and the thin-dashed curves. In a small magnetic field, most of the oscillator strength is contained in the first peak. As the magnetic field increases, the oscillator strength of the first peak is transfered to the other three.
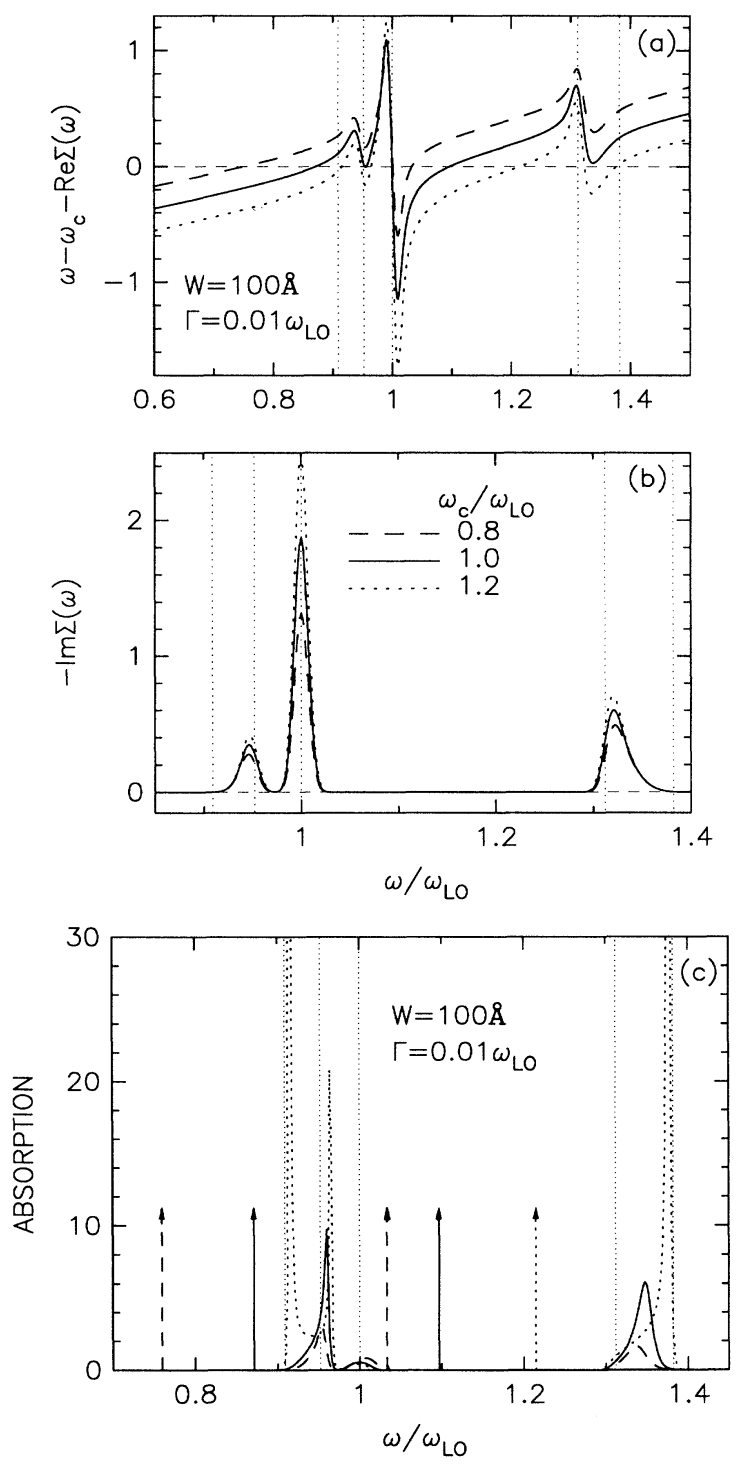

FIG. 9. (a) $\omega-\omega_{c}-\operatorname{Re} \Sigma(\omega)$ and (b) $\operatorname{Im} \Sigma(\omega)$ are plotted as a function of the frequency $\omega$ for a 100 - quantum well in different magnetic fields. The corresponding absorption spectrum is given in (c). The Landau-level broadening parameter is $\Gamma / \omega_{\mathrm{LO}}=0.01$. The vertical thin dotted lines indicate the energies $\hbar \omega_{T 1}, \hbar \omega_{-}^{\infty}, \hbar \omega_{L 1}, \hbar \omega_{+}^{\infty}$, and $\hbar \omega_{L 2}$. 
The second and the third peak reach to a maximum and then decrease. The oscillator strength of the fourth peak increases monotonously. In very high magnetic fields the fourth peak contains most of the oscillator strength. It is found that the absorption of the second peak is very weak except for narrow wells. With increasing well width the
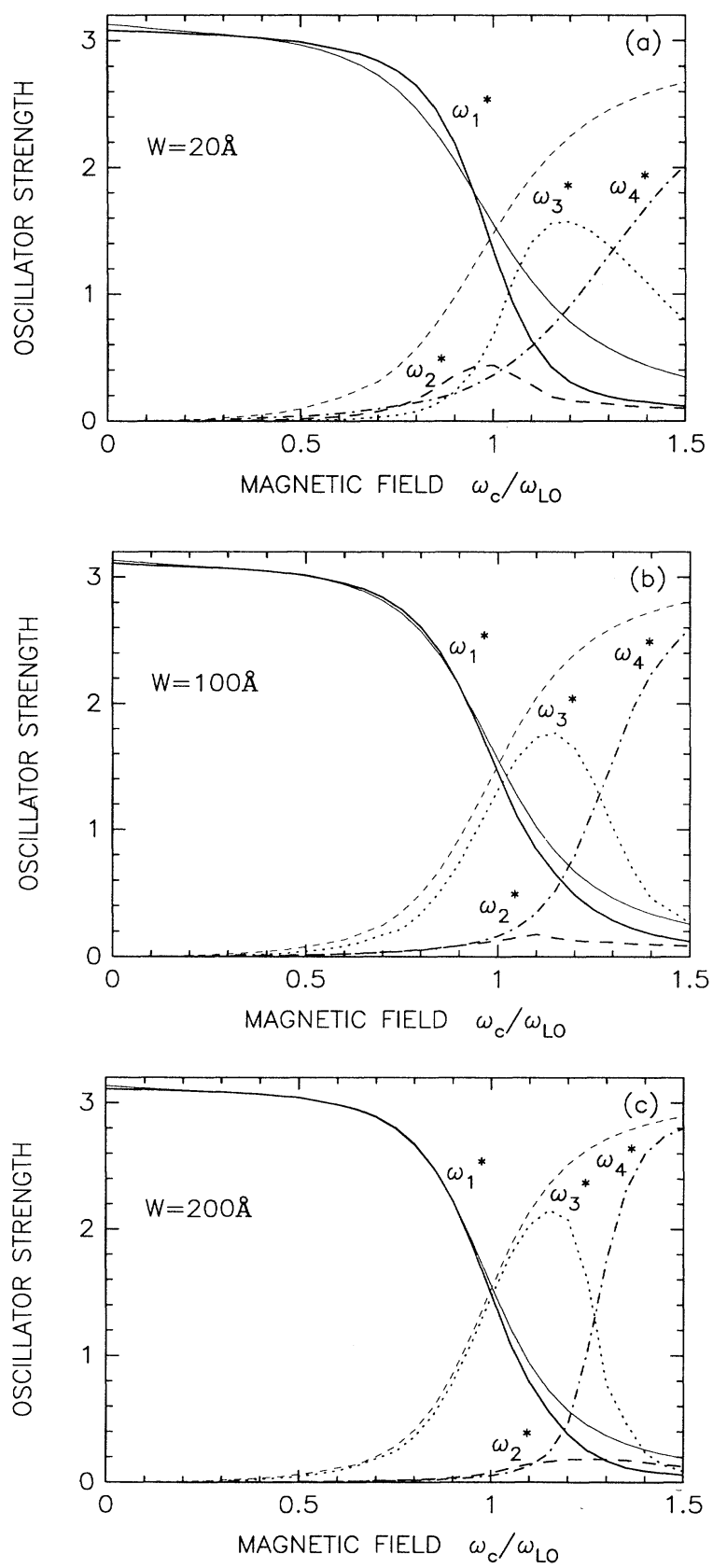

FIG. 10. The oscillator strength of the four peaks in the magneto-optical absorption spectrum as a function of the magnetic field for (a) 20-, (b) 100-, and (c) 200- $\AA$ GaAs/AlAs quantum wells with $\Gamma / \hbar \omega_{\mathrm{LO}}=0.01$. The thin solid and dashed curves are the results when only the bulk LO phonons of GaAs were included.

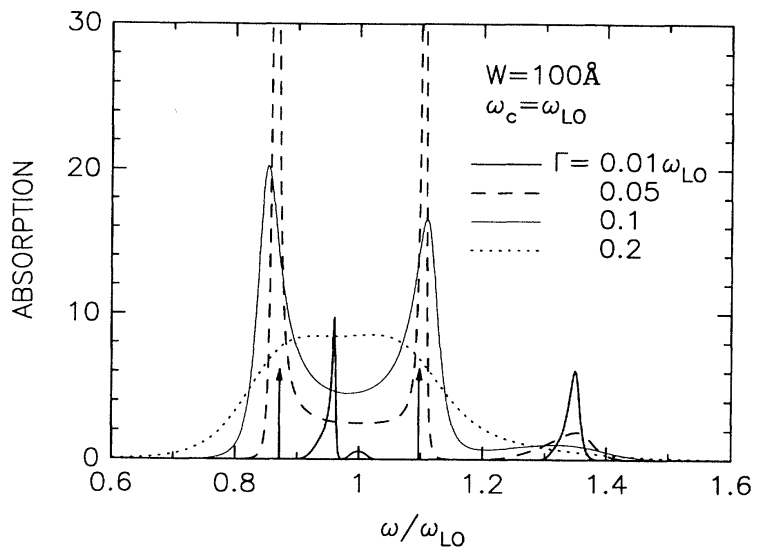

FIG. 11. The magneto-optical absorption spectrum for a $100-\AA$ quantum well with different Landau-level broadening parameters $\Gamma / \hbar \omega_{\mathrm{LO}}=0.01,0.05,0.1$, and 0.2 at $\omega_{c}=\omega_{\mathrm{LO}}$.

maximum of $\omega_{3}^{*}$ becomes larger, and the oscillator strength of the fourth peak increases more rapidly.

From Fig. 10 we see that the first branch in Fig. 7 has the largest oscillator strength when $\omega_{\varepsilon} / \omega_{\mathrm{LO}}<1.035$, 1.015 , and 1.0 for $W=20,100$, and $200 \AA$, respectively. The third branch has the largest oscillator strength when the magnetic field is in the range $1.32>\omega_{c} / \omega_{\mathrm{LO}}>1.035$, $1.28>\omega_{c} / \omega_{\mathrm{LO}}>1.015$, and $1.275>\omega_{c} / \omega_{\mathrm{LO}}>1.0$ for $W=20,100$, and $200 \AA$, respectively. And the fourth branch has the largest oscillator strength when the magnetic field is outside the above range.

So far, we have only studied the absorption spectrum with small broadening parameter $\Gamma=0.01 \omega_{\text {LO }}$. The Landau-level broadening also influences the spectrum structure. As is shown in Fig. 11 for a $100-\AA$ GaAs/AlAs quantum well at $\omega_{c}=\omega_{\mathrm{LO}}$. The thick solid curve is the spectrum with $\Gamma=0.01 \omega_{\mathrm{LO}}$ and the upward arrows indicate $\delta$-function absorption. At this moment,

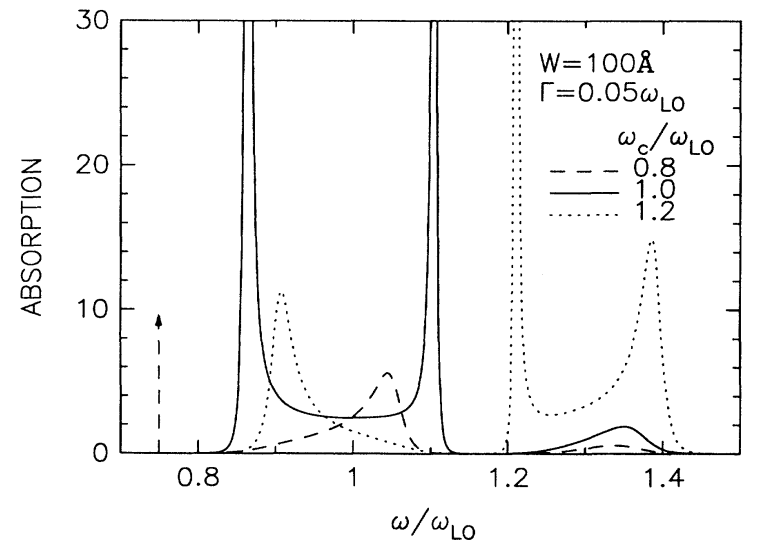

FIG. 12. The magneto-optical absorption spectrum with $\Gamma / \hbar \omega_{\mathrm{LO}}=0.05$ in different magnetic fields for a quantum well with width $W=100 \AA$. 
there are four distinct peaks. The first and the third are given by $\delta$ functions. The second and the fourth are broad with nonzero linewidths. When $\Gamma=0.05 \omega_{\text {LO }}$, the spectrum (dashed curve) reduces to three broad peaks. As $\Gamma$ increases further, the absorption peaks become broader and their maximum value reduces. When $\Gamma=0.2 \omega_{\mathrm{LO}}$, all the peaks are mixed and the absorption spectrum reduces to one broad peak.

Figure 12 gives the absorption spectrum with broadening $\Gamma=0.05 \omega_{\text {LO }}$ in different magnetic fields for a $100-\AA$ quantum well. Notice that there are only three peaks in the absorption spectrum. A comparison of the cyclotron-resonance spectrum with $\Gamma=0.01 \omega_{\text {LO }}$ and $\Gamma=0.05 \omega_{\mathrm{LO}}$ is presented in Figs. 13(a) for $W=20 \AA$, (b) for $W=100 \AA$, and (c) for $W=200 \AA$. For a Landaulevel broadening parameter $\Gamma=0.05 \omega_{\text {LO }}$ the cyclotron frequency only splits into three branches. In high magnetic fields the pinning behavior of the first branch is mainly determined by the $S$ - mode. The lower part of the second branch depends mainly on the
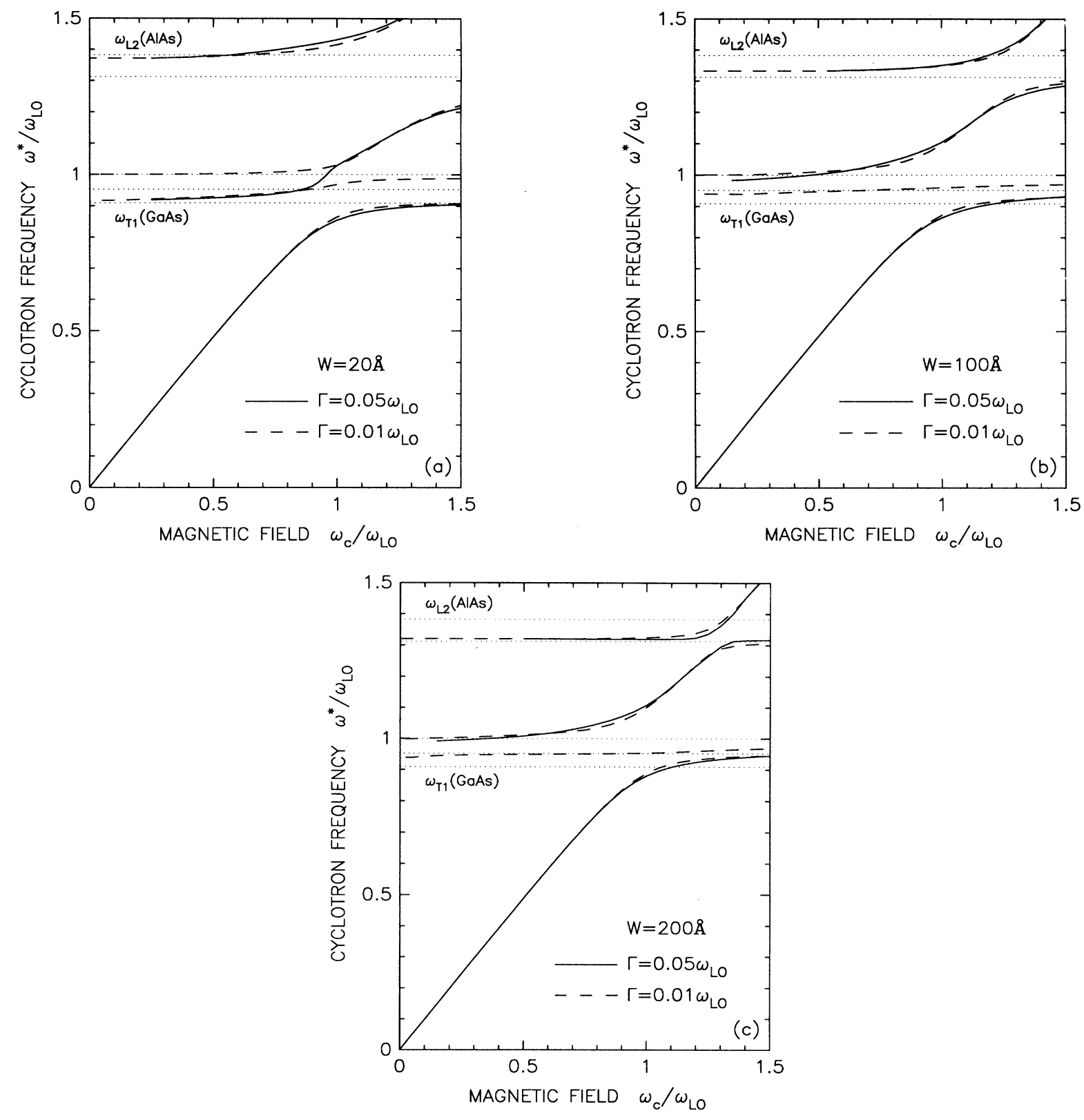

FIG. 13. The cyclotron frequency as a function of the magnetic field for (a) 20-, (b) 100-, and (c) 200- $\AA$ GaAs/AlAs quantum wells with $\Gamma / \hbar \omega_{\mathrm{LO}}=0.01$ (dashed curves) and $\Gamma / \hbar \omega_{\mathrm{LO}}=0.05$ (solid curves). 
electron-slab-LO-phonon interaction. The splitting in higher magnetic fields is due to the $S+$ interface mode and is nearly the same for the two different considered $\Gamma$.

\section{CONCLUSIONS}

In this paper we present a detailed investigation of the effect of interface optical-phonon and confined slab LOphonon modes on the polaron Landau levels and the magneto-optical absorption spectrum. We applied our theoretical results to the case of a GaAs/AlAs quantum well. As a comparison, the polaron Landau levels and the cyclotron-resonance spectrum are also calculated when only 3D LO-phonon modes of GaAs are included.

The polaron Landau levels and the cyclotron-resonant mass as a function of the magnetic field and of the well width were obtained. For an infinite-barrier well, the polaron energy and cyclotron mass vary from the 2D results at $W=0$ to the $3 \mathrm{D}$ results when $W \rightarrow \infty$ in different magnetic fields. For a finite-barrier well the results with the leading-term approximation are obtained. As a function of the magnetic field, the binding energy of the $n=0$ Landau level increases slightly with increasing magnetic field. For interface- and slab-phonon modes the $n=1$ polaron Landau level splits around the LO-phonon energy, and also around the interface-phonon energies. The interface-phonon mode plays an important role in the magnetophonon resonance in a quantum well. In a narrow well, the splitting of the Landau level around the interface-phonon energy is larger than that at the GaAs LO-phonon energy, and with increasing well width the splitting due to the interface-phonon energy decreases while that around the slab-phonon energy increases. When $W \rightarrow \infty$, they approach the result of the $3 \mathrm{D}$ system.

In order to assess the relative importance of the different resonances we investigated the magneto-optical absorption spectrum. The magnetophonon resonance occurs at three places, two of them around the $S-$ and $S+$ interface-mode frequencies, and another at the slabmode frequency. Including interface and slab phonons, the cyclotron spectrum splits into four branches. In high magnetic fields, the first three branches are pinned and the fourth approaches the unperturbed transition energy.
Furthermore, we found that the pinning position due to the interface-phonon modes depends on the width of the quantum well. For the $S-$ mode the pinning position starts from $\omega_{T 1}$ in a narrow well and reaches the $\omega_{S,-}\left(q_{\|} \rightarrow \infty\right)$ in a wide well. But, for the $S+$ mode, the pinning position decreases as the well width increases. It is at $\omega_{L 2}$ in a narrow well and at $\omega_{S+}\left(q_{\|} \rightarrow \infty\right)$ in a wide well.

In the calculation of the magneto-optical absorption spectrum, a Landau-level broadening parameter $\Gamma$ was introduced phenomenologically. The absorption spectrum structure is also influenced by the Landau-level broadening. With $\Gamma=0.05 \omega_{\text {LO }}$ we found that the four branches of the cyclotron-resonance spectrum collapse to three. Especially, the splitting due to the slab phonons disappears in a $20-\AA$ quantum well.

Our result with the $3 \mathrm{D}$ phonon modes is consistent with that from Ref. 33 in which Wu, Peeters, and Devreese had calculated the cyclotron mass in a quantum well. The present result is different from those in Refs. 47 and 48 . In Ref. 47 interface- and slab-phonon modes were included and Gu, Kong, and Wei calculated the lowest polaron Landau level associated with the first two electric levels in a $\mathrm{GaAs} / \mathrm{Al}_{x} \mathrm{Ga}_{1-x}$ As quantum well. Their result shows that, as the magnetic field increases, the polaron correction to the lowest Landau levels increases abruptly at $B \geq 10 \mathrm{~T}$. The latter is not found in our more thorough calculation. In Ref. 48 Lin, Chen, and George studied the bound polaron with interfaceand slab-phonon modes in a GaAs $/ \mathrm{Al}_{x} \mathrm{Ga}_{1-x} \mathrm{As}$ quantum well. It was claimed that the $1 s-2 p^{+}$transition is pinned to the TO-phonon frequency due to the electron-interface-phonon interaction. From the present theory for free electrons it is difficult to understand such a behavior.

\section{ACKNOWLEDGMENTS}

One of us (F.M.P.) was supported by the Belgian National Science Foundation. This work was sponsored by Fonds voor Kollektief Fundamenteel Onderzoek, Belgium, Project No. 2.0093.91 and by "Diensten voor de Programmatie van het Wetenschapsbeleid" (Belgium) under Contract No. IT/SC/24.
*Also at RUCA, B-2020 Antwerpen, Belgium, and Technical University Eindhoven, The Netherlands.

${ }^{1}$ Polarons in Ionic Crystals and Polar Semiconductors, edited by J. T. Devreese (North-Holland, Amsterdam, 1972); Polarons and Excitons in Polar Semiconductors and Ionic Crystals, edited by J. T. Devreese and F. M. Peeters (Plenum, New York, 1984); T. K. Mitra, A. Chatterjee, and S. Mukhopadhyay, Phys. Rep. 153, 91 (1987).

${ }^{2}$ D. Larsen, Phys. Rev. 135, A149 (1964); in Polarons in Ionic Crystals and Polar Semiconductors (Ref. 1), p. 237.

${ }^{3}$ R. Evrard, E. Kartheuser, and J. T. Devreese, Phys. Status Solidi B 41, 431 (1970); E. Kartheuser, R. Evrard, and J. T. Devreese, Solid State Commun. 10, 15 (1972); J. T. Devreese, E. Kartheuser, R. Evrard, and A. Baldereschi, Phys. Status
Solidi B 59, 629 (1973).

${ }^{4}$ K. K. Bajaj, Phys. Rev. 170, 694 (1968); Solid State Commun. 8, 1907 (1970).

${ }^{5}$ F. M. Peeters and J. T. Devreese, Solid State Commun. 39, 445 (1981); Phys. Rev. B 25, 7281 (1982); 25, 7302 (1982).

${ }^{6}$ Y. Lépine and D. Matz, Can. J. Phys. 54, 1979 (1976); Y. Lépine, J. Phys. C 18, 1817 (1985).

${ }^{7}$ E. J. Johnson and D. M. Larsen, Phys. Rev. Lett. 16, 655 (1966).

${ }^{8}$ R. Kaplan and R. F. Wallis, Phys. Rev. Lett. 20, 1499 (1968).

${ }^{9}$ B. D. McCombe and R. Kaplan, Phys. Rev. 21, 756 (1968).

${ }^{10} \mathrm{G}$. Lindemann, R. Lassnig, W. Seidenbusch, and E. Gornik, Phys. Rev. B 28, 4639 (1983).

${ }^{11}$ J. W. Hodby, G. P. Russell, F. M. Peeters, J. T. Devreese, and 
D. M. Larsen, Phys. Rev. Lett. 58, 1471 (1987).

${ }^{12}$ F. M. Peeters and J. T. Devreese, Phys. Rev. B 34, 7246 (1986).

${ }^{13}$ X.-G. Wu, F. M. Peeters, and J. T. Devreese, Phys. Rev. B 31, 3420 (1985).

${ }^{14} \mathrm{~J}$. T. Devreese and F. M. Peeters, in The Physics of the TwoDimensional Electron Gas, edited by J. T. Devreese and F. M. Peeters (Plenum, New York, 1987), p. 131.

${ }^{15}$ S. Das Sarma and A. Madhukar, Phys. Rev. B 22, 2823 (1980).

${ }^{16}$ S. Das Sarma, Phys. Rev. B 27, 2590 (1983).

${ }^{17}$ S. Das Sarma and M. Stopa, Phys. Rev. B 36, 9595 (1987)

${ }^{18}$ F. M. Peeters and J. T. Devreese, Phys. Rev. B 36, 4442 (1987).

${ }^{19}$ X.-G. Wu, F. M. Peeters, and J. T. Devreese, Phys. Rev. B 34, 8800 (1986)

${ }^{20}$ F. M. Peeters and J. T. Devreese, Phys. Rev. B 31, 3689 (1985).

${ }^{21}$ F. M. Peeters, X.-G. Wu, and J. T. Devreese, Phys. Rev. B 33, 4338 (1986).

${ }^{22}$ F. M. Peeters, X.-G. Wu, and J. T. Devreese, Phys. Scr. T13, 282 (1986).

${ }^{23}$ D. M. Larsen, Phys. Rev. B 30, 4595 (1984); 30, 4807 (1984).

${ }^{24}$ X.-G. Wu, F. M. Peeters, and J. T. Devreese, Phys. Status Solidi B 133, 229 (1986).

${ }^{25}$ F. M. Peeters, X.-G. Wu, J. T. Devreese, C. J. G. M. Langerak, J. Singleton, D. J. Barnes, and R. J. Nicholas, Phys. Rev. B 45, 4296 (1992).

${ }^{26}$ M. Horst, U. Merkt, and J. P. Kotthaus, Phys. Rev. Lett. 50 754 (1983).

${ }^{27}$ J. Scholz, F. Koch, J. Ziegler, and H. Maier, Solid State Commun. 46, 665 (1983).

${ }^{28}$ M. Horst, U. Merkt, W. Zawadzki, J. C. Maan, and R. Ploog, Solid State Commun. 53, 403 (1985).

${ }^{29}$ H. Sigg, P. Wyder, and J. A. A. J. Perenboom, Phys. Rev. B 31, 5253 (1985).

${ }^{30}$ W. Seidenbusch, G. Lindemann, R. Lassnig, J. Edlinger, and
E. Gornik, Surf. Sci. 142, 375 (1984).

${ }^{31}$ M. Zeismann, D. Heitmann, and L. L. Chang, Phys. Rev. B 35, 4541 (1987).

32J. Singleton, R. J. Nicholas, D. C. Rogers, and C. T. B. Foxon, Surf. Sci. 196, 429 (1988).

${ }^{33}$ X.-G. Wu, F. M. Peeters, and J. T. Devreese, Phys. Rev. B 40, 4090 (1989).

${ }^{34}$ L. Wendler, Phys. Status Solidi B 129, 513 (1985).

${ }^{35}$ L. Wendler and R. Pechstedt, Phys. Status Solidi B 141, 129 (1987).

${ }^{36}$ N. Mori and T. Ando, Phys. Rev. B 40, 6175 (1989).

${ }^{37}$ K. Haung and B. F. Zhu, Phys. Rev. B 38, 13377 (1988).

38 J. J. Licari and R. Evrard, Phys. Rev. B 15, 2254 (1977).

${ }^{39}$ J. J. Lacari, Solid State Commun. 29, 625 (1979).

${ }^{40}$ X. X. Liang, S. W. Gu, and D. L. Lin, Phys. Rev. B 34, 2807 (1986).

${ }^{41}$ F. Comas, C. Trallero-Giner, and R. Riera, Phys. Rev. B 39, 5907 (1989).

${ }^{42}$ M. H. Degani and O. Hipólito, Phys. Rev. B 35, 7717 (1987).

${ }^{43}$ M. H. Degani and O. Hipólito, Superlatt. Microstruct. 5, 141 (1989).

${ }^{44}$ G. Q. Hai, F. M. Peeters, and J. T. Devreese, Phys. Rev. B 42, 11063 (1991).

${ }^{45}$ S. W. Gu, X. J. Kong, and C. W. Wei, J. Phys. C 21, 1497 (1988).

${ }^{46}$ X. X. Liang, Phys. Rev. B 38, 3459 (1988).

${ }^{47}$ S. W. Gu, X. J. Kong, and C. W. Wei, Phys. Rev. B 36, 7984 (1987).

${ }^{48}$ D. L. Lin, R. Chen, and T. F. George, Phys. Rev. B 43, 9328 (1991).

${ }^{49}$ S. Adachi, J. Appl. Phys. 58, R1 (1985).

${ }^{50}$ F. M. Peeters and J. T. Devreese, Phys. Rev. B 23, 1936 (1981).

${ }^{51}$ F. M. Peeters and J. T. Devreese, Phys. Rev. B 28, 6501 (1983). 